\title{
INCLUSIONS AND NONINCLUSION OF SPACES OF CONVOLUTION OPERATORS
}

\author{
BY \\ MICHAEL G. COWLING AND JOHN J. F. FOURNIER( ${ }^{(1)}$
}

ABSTRACT. Let $G$ be an infinite, locally compact group. Denote the space of convolution operators, on $G$, of strong type $(p, q)$ by $L_{p}^{q}(G)$. It is shown that, if $|1 / q-1 / 2|<|1 / p-1 / 2|$, then $L_{q}^{q}(G)$ is not included in $L_{p}^{p}(G)$. This result follows from estimates on the norms, in these spaces, of Rudin-Shapiro measures. The same method leads to a simple example of a convolution operator that is of strong type $(q, q)$ for all $q$ in the inter$\operatorname{val}\left(p, p^{\prime}\right)$ but is not of restricted weak type $(p, p)$ or of restricted weak type $\left(p^{\prime}, p^{\prime}\right)$. Other statements about noninclusion among the spaces $L_{p}^{q}(G)$ also follow from various assumptions about $G$. For instance, if $G$ is unimodular, but not compact, $1<p, q, r, s<\infty$, with $p<q$, and $\min \left(s, r^{\prime}\right)<\min \left(q, p^{\prime}\right)$, then $L_{p}^{q}(G)$ is not included in $L_{r}^{s}(G)$.

Using Zafran's multilinear interpolation theorem for the real method, it is shown that, if $1<p<2$, then there exists a convolution operator on $G$ that is of weak type $(p, p)$ but not of strong type $(p, p)$; it is not known whether such operators exist when $p>2$, but it is shown that if $p \neq 1,2, \infty$, then there exists a convolution operator that is of restricted weak type $(p, p)$ but is not of weak type $(p, p)$.

Many of these results also hold for the spaces of operators that commute with left translation rather than right translation. Further refinements are presented in three appendices.

1. Introduction. Throughout the paper, we let $G$ denote a locally compact group, and we write the group operation multiplicatively. For an element $a$ of $G$ we denote the unit point mass at $a$ by $\epsilon(a)$; if $f$ is an object on $G$ such that the convolutions $\epsilon(a) * f$ and $f *[\epsilon(a)]$ are defined, then we refer to these products as the left and right translates of $f$ by $a$, and we also denote then by $L(a) f$ and $R(a) f$, respectively. By a convolution operator on $G$, we mean a linear operator, from one space of functions on $G$ that is closed under right translation to another such space, that commutes with right translation; that is, $T$ is a convolu-

Received by the editors April 14, 1975.

AMS (MOS) subject classifications (1970). Primary 43A22, 42A18, 46E35; Secondary 43A15, 46E30.

Key words and phrases. Locally compact group, convolution operator, strong type, weak type, noninclusion, real interpolation method, complex interpolation method, RudinShapiro measures.

(1) Research partially supported by Canadian NRC grant number 4822 . 
tion operator if $T(R(a) f)=R(a)(T f)$ for all $a$ in $G$, and all $f$ in the domain of $T$. Some authors use terminology such as centralizer, convoluteur, or multiplier operator for what we call a convolution operator.

Let $m$ be a left-invariant Haar measure on $G$, and form the usual Lebesgue spaces $L^{p}(G)$ with respect to $m$; as usual, we refer to members of $L^{p}(G)$ as functions although they are really equivalence classes of functions. Denote the norm on $L^{p}(G)$ by $\|\cdot\|_{p}$. Let $C_{c}(G)$ be the space of all continuous functions on $G$ with compact support. For $p$ and $q$ in the interval $[1, \infty]$, we let $L_{q}^{p}(G)$ be the space of all convolution operators $T$, with domain $C_{c}(G)$, for which there exists a constant $D$ such that

$$
\|T f\|_{q} \leqslant D\|f\|_{p} \text { for all } f \text { in } C_{c}(G) .
$$

Equivalently, we can regard $L_{p}^{q}(G)$ as the space of all bounded linear operators, from the closure of $C_{c}(G)$ in $L^{p}(G)$ to $L^{q}(G)$, that commute with right translations; we then give $L_{p}^{q}(G)$ the operator norm, making it a Banach space.

An operator $T$ satisfying inequality (1) is said to be of strong type $(p, q)$. If $q<\infty$, then $T$ is said to be of weak type $(p, q)$ provided there exists a constant $D$ such that

$$
m\{x \in G:|T f(x)|>\lambda\} \leqslant\left(D\|f\|_{p} / \lambda\right)^{q},
$$

for all $\lambda>0$ and all $f$ in the domain of $T$. Finally, $T$ is said to be of restricted, weak type $(p, q)$ if inequality (2) holds whenever $\lambda>0$ and $f$ is the characteristic function of a set of finite measure.

Many important convolution operators in harmonic analysis are of weak type $(1,1)$ but not of strong type $(1,1)$; see [Ste 3] for examples. Simple examples are given in [SW 1], for $1<p<\infty$, of operators that are of weak type $(p, p)$ but not of strong type $(p, p)$; these operators, however, are not convolution operators. If the group $G$ is amenable, then every convolution operator of weak type $(2,2)$ is in fact of strong type $(2,2)$. It was conjectured that the same might be true with the index 2 replaced by any $p$ in the interval $(1,2)$, but Misha Zafran recently showed [Zaf 2], for various infinite, locally compact, abelian groups, that, for each $p$ in the interval $(1,2)$, there exists a convolution operator that is of weak type $(p, p)$ but not of strong type $(p, p)$. One of the main results of the present paper is that such convolution operators exist on any infinite, locally compact group.

This result is proved in §3. Two key elements in the proof are Zafran's multilinear interpolation theorem for the real method, and some estimates on the norms, in the spaces $L_{p}^{p}(G)$, of Rudin-Shapiro measures. These estimates are obtained in $\S 2$, where they are used to give a quick proof that, if $G$ is infinite, and $|1 / q-1 / 2|<|1 / p-1 / 2|$, then $L_{q}^{q}(G)$ is not included in $L_{p}^{p}(G)$. In $\S 4$, we 
use similar methods to obtain noninclusion theorems for the spaces $L_{p}^{q}(G)$ with $p \neq q$.

$\S 5$ concerns inclusions between spaces of operators that commute with left translation rather than right translation. If $G$ is unimodular, then the same inclusions hold for these spaces as for the spaces $L_{p}^{q}(G)$. We show, however, that if $G$ is not unimodular, then there are no inclusions between these spaces; we also consider inclusion relations for modified versions of these spaces, and we then recover analogues of the results that hold for the spaces $L_{p}^{q}(G)$.

We have relegated some results of three appendices, because the statements of these results are complicated, or because their proofs have little in common with the methods used in the rest of the paper. In Appendix 1, we consider relations between the structure of $G$ and and the inclusion properties of the spaces $L_{p}^{q}(G)$; a typical result is that, if $G$ is a near-connected, unimodular, locally compact group, and if $L_{p}^{q}(G) \subset L_{r}^{s}(G)$ for indices $p, q, r, s$ with $1 \leqslant p \leqslant q \leqslant \infty$, and $1 / p-1 / q>1 / r-1 / s$, then $G$ has a compact, normal subgroup $N$ such that $G / N$ is a semisimple Lie group, with finite centre. In Appendix 2, we consider inclusions between the spaces of convolution operators that map one Lorentz space into another Lorentz space; we show that, if $G$ is infinite, and $p \neq 1,2, \infty$, then there exists a convolution operator of restricted weak type $(p, p)$ that fails to map $L^{p, q}(G)$ into $L^{p, r}(G)$ whenever $q>1$ or $r<\infty$. Finally, in Appendix 3, we offer a generalization to noncompact groups of the known fact that, if $G$ is compact, and $1 \leqslant p<2$, then all the spaces $L_{p}^{q}(G)$ with $q<p$ coincide with the space of convolution operators of weak type $(p, p)$.

2. Rudin-Shapiro measures. If $\mu$ is a finite, regular, Borel measure, with total variation $\|\mu\|$, and if $f \in L^{p}(G)$, then the convolution $\mu * f$ belongs to $L^{p}(G)$, and

$$
\|\mu * f\|_{p} \leqslant\|\mu\|\|f\|_{p}
$$

[HR, 20.12]. Thus, we can identify $\mu$ with the operator $f \mapsto \mu * f$ which belongs to $L_{p}^{p}(G)$ and has norm at most $\|\mu\|$. In this section, we construct RudinShapiro measures on any infinite group, and we estimate their norms in the spaces $L_{p}^{p}(G)$. Using these estimates, we show that, if $G$ is infinite, and $\mid 1 / q-$ $1 / 2|<| 1 / p-1 / 2 \mid$, then $L_{q}^{q}(G)$ is not included in $L_{p}^{p}(G)$. In fact, for each index $p$ in the interval $(1,2)$, we give an explicit example of a convolution operator that is of strong type $(q, q)$ for all $q$ in the interval $\left(p, p^{\prime}\right)$, but is not of restricted weak type $(p, p)$ or of restricted weak type $\left(p^{\prime}, p^{\prime}\right)$; here $p^{\prime}$ denotes the index conjugate to $p$.

Rudin-Shapiro polynomials have been used by Gaudry [Gau] to prove theorems about noninclusions of spaces of convolution operators on abelian groups. Rudin-Shapiro measures have been constructed on the circle group by 
Kahane [Kah, pp. 34-36], and used in the study of the Fourier algebra of that group.

Before constructing such measures on any infinite group, we recall some properties of left and right translation. First, for any function $f$, and all elements $a$ and $x$ of $G$,

$$
(L(a) f)(x)=f\left(a^{-1} x\right)
$$

thus $L(a)$ is an isometry on $L^{p}(G)$ for all $p$. Also, if $\mu$ is a Borel measure and $E$ is a Borel subset of $G$, then

$$
(L(a) \mu)(E)=\mu\left(a^{-1} E\right) .
$$

The corresponding descriptions of right translation are more complicated in general. Let $\Delta$ denote the modular function on $G$. Then

$$
(R(a) f)(x)=\Delta\left(a^{-1}\right) f\left(x a^{-1}\right)
$$

[HR, 20.9]. This means that, if $\mu$ is absolutely continuous, then

$$
(R(a) \mu)(E)=\Delta\left(a^{-1}\right) \mu\left(E a^{-1}\right) .
$$

On the other hand, if $\mu$ is discrete, then

$$
(R(a) \mu)(E)=\mu\left(E a^{-1}\right)
$$

one way to see this is to regard the convolution $\mu *[\epsilon(a)]$ as being carried out on the group made discrete and hence unimodular.

We now suppose that $G$ is infinite, and we inductively construct RudinShapiro measures. First, let $\rho_{0}$ and $\sigma_{0}$ both be $\epsilon(e)$, the unit point mass at the identity. Suppose that $\rho_{n}$ and $\sigma_{n}$ have been defined and are discrete measures both supported by a finite set $S_{n}$; then choose an element $x_{n}$ of $G$ so that the sets $x_{n} S_{n}$ and $S_{n}$ are disjoint, and let

$$
\rho_{n+1}=\rho_{n}+L\left(x_{n}\right) \sigma_{n},
$$

and

$$
\sigma_{n+1}=\rho_{n}-L\left(x_{n}\right) \sigma_{n}
$$

It is easy to verify by induction on $n$ that there are a sequence $\left(y_{k}\right)_{k=1}^{\infty}$ of distinct points of $G$, and fixed \pm 1 -valued sequences $\left(r_{k}\right)_{k=1}^{\infty}$ and $\left(s_{k}^{n}\right)^{\infty}=1$ such that

$$
\rho_{n}=\sum_{k=1}^{2^{n}} r_{k} \epsilon\left(y_{k}\right)
$$

and

$$
\sigma_{n}=\sum_{k=1}^{2^{n}} s_{k}^{n} \epsilon\left(y_{k}\right)
$$


We now estimate the norms of these measures in the spaces $L_{p}^{p}(G)$.

LeMmA 1. Let $1 \leqslant p \leqslant \infty$, and let $r=\max \left(p, p^{\prime}\right)$. Then

$$
2^{n / r} \leqslant\left\|\rho_{n}\right\|_{L p} \leqslant \sqrt{2} 2^{n / r}
$$

and

$$
2^{n / r} \leqslant\left\|\sigma_{n}\right\|_{L p} \leqslant \sqrt{2} 2^{n / r} .
$$

Proof. The key property of these measures is that, if $f \in C_{c}(G)$, then

$$
\left\|\rho_{n} * f\right\|_{2}^{2}+\left\|\sigma_{n} * f\right\|_{2}^{2}=2^{n+1}\|f\|_{2}^{2}
$$

This clearly holds if $n=0$. On the other hand, by the parallelogram law,

$$
\begin{aligned}
\left\|\rho_{n+1} * f\right\|_{2}^{2}+\left\|\sigma_{n+1} * f\right\|_{2}^{2} & =2\left(\left\|\rho_{n} * f\right\|_{2}^{2}+\left\|L\left(x_{n}\right) \sigma_{n} * f\right\|_{2}^{2}\right) \\
& =2\left(\left\|\rho_{n} * f\right\|_{2}^{2}+\left\|\sigma_{n} * f\right\|_{2}^{2}\right),
\end{aligned}
$$

and relation (10) follows by induction on $n$. Now, by inequality (3),

$$
\left\|\rho_{n}\right\|_{L_{1}^{1}} \leqslant\left\|\rho_{n}\right\|=2^{n} \text { and }\left\|\rho_{n}\right\|_{L_{\infty}^{\infty}} \leqslant 2^{n}
$$

while, by relation (10),

$$
\left\|\rho_{n}\right\|_{L_{2}^{2}} \leqslant 2^{(n+1) / 2} .
$$

Therefore, $\left\|\rho_{n}\right\|_{L p} \leqslant \sqrt{2} 2^{n / r}$ if $p=1,2$, or $\infty$. But the Riesz convexity theorem [SW 2, p. 179] states that $\left\|\rho_{n}\right\|_{L} p$ is a logarithmically convex function of $1 / p$, and it follows that $\left\|\rho_{n}\right\|_{L} p \leqslant \sqrt{2} 2^{n / r}$ for all $p$.

To get the lower bound on the norm of $\rho_{n}$, we observe that, if $f$ is supported by a sufficiently small open set, then the functions $\epsilon\left(y_{k}\right) * f$, for $k=$ $1,2, \ldots, 2^{n}$, have disjoint support. Thus $\rho_{n} * f$ is a sum of $2^{n}$ disjointly-supported left translates of $f$, so that $\left\|\rho_{n} * f\right\|_{p}=2^{n / p}\|f\|_{p}$. This shows that $\left\|\rho_{n}\right\|_{L p} \geqslant 2^{n / p}$, which is the desired lower bound if $p \leqslant 2$. If $p>2$, we consider $\rho_{n}^{v}$, the reflection of $\rho_{n}$; that is, $\rho_{n}^{\vee}=\Sigma_{k=1}^{2 n} r_{k} \in\left(y_{k}^{-1}\right)$. Now,

$$
\int_{G}\left[\rho_{n} * f(x)\right] g(x) d x=\int_{G} f(x)\left[\rho_{n}^{\vee} * g(x)\right] d x ;
$$

that is, the adjoint of the map $f \mapsto \rho_{n} * f$ is the map $f \mapsto \rho_{n}^{\vee} * f$. Therefore, if $2<p \leqslant \infty$, then

$$
\left\|\rho_{n}\right\|_{L_{p}^{p}}=\left\|\rho_{n}^{\vee}\right\|_{L_{p^{\prime}}^{p^{\prime}}} \geqslant 2^{n / p^{\prime}}=2^{n / r}
$$

This completes the proof of statement (8). Clearly, the same arguments apply with $\sigma_{n}$ in place of $\rho_{n}$, so that statement (9) is also proved. 
Before stating our theorem about noninclusion of the spaces $L_{p}^{p}(G)$, we summarize the known inclusions of these spaces. First, by a theorem of Wendel [Wen], $L_{1}^{1}(G)=M(G)$, the space of bounded, regular Borel measures with the total variation norm; then, by inequality (3), $L_{1}^{1}(G) \subset L_{p}^{p}(G)$ for all $p$. If $G$ is abelian, then $L_{p}^{p}(G)=L_{p}^{p^{\prime}}(G)$, and $L_{p}^{p}(G) \subset L_{q}^{q}(G)$ whenever $|1 / q-1 / 2|<$ $|1 / p-1 / 2|$; see [Lar, p. 114]. In fact, these inclusions also hold if $G$ has an abelian subgroup of finite index, but Daniel Oberlin recently found a compact group $G$ for which $L_{4}^{4}(G) \neq L_{4 / 3}^{4 / 3}(G)$ [Obe]. If $G$ is amenable, then $L_{p}^{p}(G) \subset$ $L_{2}^{2}(G)\left[\right.$ Her 1], and $L_{p}^{p}(G) \subset L_{q}^{q}(G)$ whenever $|1 / q-1 / 2|<|1 / p-1 / 2|$ and $q$ is on the same side of 2 as $p$. The following theorem shows that these inclusions are strict if $G$ is infinite.

THEOREM 2. Let $G$ be an infinite, locally compact group, and let $p$ and $q$ be indices in the interval $[1, \infty]$ satisfying the inequality $|1 / q-1 / 2|<\mid 1 / p-$ $1 / 21$. Then the space $L_{q}^{q}(G)$ is not included in the space $L_{p}^{p}(G)$.

Proof. Suppose, to the contrary, that $L_{q}^{q}(G) \subset L_{p}^{p}(G)$. Then the injection $i: L_{q}^{q}(G) \rightarrow L_{p}^{p}(G)$ must have a closed graph. Indeed, if a sequence $\left(T_{n}\right)_{n=1}^{\infty}$ converges to 0 in $L_{q}^{q}(G)$, and to $T$ in $L_{p}^{p}(G)$, then, for all $f$ in $C_{c}(G)$, we have that $T_{n} f \rightarrow 0$ in measure, and that $T_{n} f \rightarrow T f$ in measure; therefore $T f=0$ for all such $f$, and $T=0$. This proves that $i$ has a closed graph, and hence, by the closed-graph theorem, that $i$ must be continuous. On the other hand, the estimates obtained in Lemma 1 show that

$$
\left\|\rho_{n}\right\|_{L} p_{p}^{p} /\left\|\rho_{n}\right\|_{L q} \rightarrow \infty \quad \text { as } n \rightarrow \infty .
$$

This contradiction proves that $L_{q}^{q}(G) \not \subset L_{p}^{p}(G)$, and completes the proof of the theorem.

We now indicate how to construct explicit examples of convolution operators that belong to $L_{q}^{q}(G)$ but not to $L_{p}^{p}(G)$. Fix $p$ in the interval $[1,2]$. Choose disjointly-supported left translates $\rho_{n}^{\prime}$ of the measures $\rho_{n}$. The estimates, on the norm of $\rho_{n}$, obtained in Lemma 1 also hold for the norm of $\rho_{n}^{\prime}$. Therefore, the series

$$
\sum_{n=0}^{\infty} 2^{-n / p} \rho_{n}^{\prime}
$$

converges in $L_{q}^{q}(G)$ whenever $|1 / q-1 / 2|<|1 / p-1 / 2|$; let $T_{0}$ denote the sum of this series and let

$$
\mu_{N}=\sum_{n=0}^{N} 2^{-n / p} \rho_{n}^{\prime} .
$$

If $T_{0} \in L_{p}^{p}(G)$, then, by Riesz convexity, $\left\|T_{0}\right\|_{L} q$ must remain bounded as $q$ 
converges to $p$ from above. But, as in the proof of Lemma 1, we see that

$$
\left\|\mu_{N}\right\|_{L q} \geqslant\left(\sum_{n=0}^{N} 2^{-n q / p} \cdot 2^{n}\right)^{1 / q}
$$

whenever $q \leqslant 2$. Hence

$$
\left\|T_{0}\right\|_{L q} \geqslant\left(\sum_{n=0}^{\infty} 2^{n(1-q / p)}\right)^{1 / q},
$$

and $\left\|T_{0}\right\|_{L} g$ does not remain bounded as $q$ approaches $p$ from above. Thus $T_{0} \notin L_{p}^{p}(G)$, and, by a similar argument, $T_{0} \notin L_{p^{\prime}}^{p^{\prime}}(G)$ also. The methods of the next section can be used to show that $T_{0}$ is of restricted weak types $(p, p)$ and $\left(p^{\prime}, p^{\prime}\right)$. Now let $\delta$ be a real parameter, and consider the series

$$
\sum_{n=0}^{\infty}(n+1)^{-\delta} 2^{-n / p} \rho_{n}^{\prime},
$$

which converges in $L_{q}^{q}(G)$ whenever $|1 / q-1 / 2|<|1 / p-1 / 2|$. Denote the sum of the series by $T_{\delta}$. If $T_{\delta}$ is of restricted weak type $(p, p)$, then by Hunt's weak-type interpolation theorem [Hun, p. 264], $\left\|T_{\delta}\right\|_{L} g=O\left((q-p)^{-1 / p}\right)$ as $q$ approaches $p$ from above. If $\delta<0$, however, then it follows, as above, that $\left\|T_{\delta}\right\|_{L q}$ grows more rapidly than $(q-p)^{-1 / p}$ as $q$ approaches $p$ from above. In this case, then, $T_{\delta}$ is not of restricted weak type $(p, p)$, and, by a similar argument, not of restricted weak type $\left(p^{\prime}, p^{\prime}\right)$. Similar results have been proved by various authors, using various methods. For instance, if $G$ is compact and infinite, and if $1<p<2$, then there exists a convolution operator on $G$ that is of strong type $(q, 2)$ for all $q$ in the interval $(p, 2)$, but is not of restricted weak type $(p, p)$ [Fou, p. 33]. If $G$ is infinite, compact, and abelian, and if $1<p$ $<2$, then there exists a convolution operator on $G$ that maps $L^{p, 2}(G)$ into $L^{2}(G)$, but which, for each $r>2$, fails to map $L^{p, r}(G)$ into $L^{1}(G)$ [Misha Zafran, private communication]. Finally, using the Fefferman-Stein theory of $H^{p}$ spaces [FS] , E. M. Stein found an example of a convolution operator on the real line that is of strong type $(q, q)$, for all $q$ in the interval $\left(p, p^{\prime}\right)$, but not of restricted weak type $(p, p)$ [grapevine]. The series (13) and (15) are related to those used for similar purposes in [Kat, p. 34] and [Zaf 2].

REMARK 1. We can arrange matters so that the set $\left\{y_{k}\right\}_{k=1}^{\infty}$, which supports the measures $\rho_{n}$ and $\sigma_{n}$, has various convenient properties. For instance, on the circle group, the $y_{k}$ can be chosen to lie in a given, symmetric Cantor set [Kah, p. 35]. If $G$ is not compact, then, given a compact neighbourhood $V$ of the identity, the $y_{k}$ can be chosen so that the sets $y_{k} V$ are disjoint, as are the sets $V y_{k}$. Finally, even if $G$ is not unimodular, the $y_{k}$ can be chosen to lie in the kernel of the modular function, because if $G$ is not unimodular, this kernel is not compact [HR, 38.26]. 
3. Convolution operators of weak type. In this section, we show that if $G$ is an infinite, locally compact group, and if $1<p<2$, then there exists a convolution operator on $G$ that is of weak type $(p, p)$ but is not of strong type $(p, p)$. Two key elements in the proof are the Rudin-Shapiro measures constructed in the previous section, and Zafran's multilinear interpolation theorem for the real method of Lions and Peetre. Standard references concerning this method are [BB], [LP], and [Pee]. The intermediate spaces can be defined in several, equivalent ways; we use the $K$-method of Peetre.

Let $A_{0}$ and $A_{1}$ be two Banach spaces, both continuously imbedded in some hausdorff, topological vector space $V$; then $A_{0}$ and $A_{1}$ are said to form an interpolation pair. Identify $A_{0}$ and $A_{1}$ with their images in $V$, so that the spaces $A_{0} \cap A_{1}$ and $A_{0}+A_{1}$ are well defined. Let $\|\cdot\|_{0}$ and $\|\cdot\|_{1}$ denote the norms in $A_{0}$ and $A_{1}$ respectively. For each element $f$ of $A_{0}$ and $A_{1}$, and each positive real number $t$, let

$$
K(t ; f)=\inf \left\{\left\|f_{0}\right\|_{0}+t\left\|f_{1}\right\|_{1}: f=f_{0}+f_{1}, f_{0} \in A_{0}, f_{1} \in A_{1}\right\}
$$

then $K(\cdot ; f)$ is a nondecreasing function on the interval $(0, \infty)$. Fix parameters $\theta$ and $q$ with $0<\theta<1$, and $1 \leqslant q \leqslant \infty$; let $L_{*}^{q}$ denote the space of functions on $(0, \infty)$ that are in $L^{q}$ with respect to the measure $d t / t$. Define the intermediate space $\left(A_{0}, A_{1}\right)_{\theta, q}$ to consist of all elements $f$ of $A_{0}+A_{1}$ for which the map $t \mapsto t^{-\theta} K(t ; f)$ belongs to $L_{*}^{q}$. Define a norm on this intermediate space by letting

$$
\|f\|_{\theta, q}=\left\{\int_{0}^{\infty}\left[t^{-\theta} K(t ; f)\right]^{q} \frac{d t}{t}\right\}^{1 / q}, \text { if } q<\infty,
$$

and letting $\|f\|_{\theta, \infty}=\sup _{t} t^{-\theta} K(t ; f)$. Relative to this norm, $\left(A_{0}, A_{1}\right)_{\theta, q}$ is a Banach space. Clearly, $\left(A_{0}, A_{1}\right)_{\theta, q} \subset A_{0}+A_{1}$; it can also be verified that $A_{0} \cap A_{1} \subset\left(A_{0}, A_{1}\right)_{\theta, q}$.

The relevance of this machinery to the problem that we are considering becomes clear when we take $A_{0}$ and $A_{1}$ to be the spaces $L^{p_{0}}(G)$ and $L^{p_{1}}(G)$, for distinct indices $p_{0}$ and $p_{1}$ in the interval $[1, \infty]$. Then, up to equivalence of norms, the intermediate space $\left(A_{0}, A_{1}\right)_{\theta, q}$ is the Lorentz space $L^{p, q}(G)$, with $1 / p=(1-\theta) / p_{0}+\theta / p_{1}[\mathrm{BB}, \mathrm{p}$. 187]. Lorentz spaces are discussed in [BB, pp. 181-187] and [Hun]. We recall some properties of these spaces. Fix $p$ in the interval $(1, \infty)$. Then $L^{p, p}(G)$ is just $L^{p}(G)$ with an equivalent norm; a function $f$ belongs to $L^{p, \infty}(G)$ if and only if there exists a constant $C$ such that $m\{x \in G$ : $|f(x)|>\lambda\} \leqslant(C / \lambda)^{p}$, for all positive $\lambda$. Also, a linear operator $T$ is bounded from $L^{p, p}(G)$ to $L^{p, \infty}$ if and only if $T$ is of weak type $(p, p)$; finally $T$ is bounded from $L^{p, 1}(G)$ to $L^{p, \infty}(G)$ if and only if $T$ is of restricted weak type $(p, p)$.

Let $1<p<\infty$, and let $1 \leqslant q, r \leqslant \infty$. Given a locally compact group $G$, 
let $L_{p, q}^{p, r}$ denote the space of convolution operators $T$, with domain $C_{c}(G)$, for which there exists a constant $D$ such that $\|T f\|_{p, r} \leqslant D\|f\|_{p, q}$ for all $f$ in $C_{c}(G)$. Here we use \|\|$_{p, s}$ to denote the norm in the Lorentz space $L^{p, s}(G)$. Equivalently, we can regard $L_{p, q}^{p, r}$ as the space of all bounded linear operators, from the closure of $C_{c}(G)$ in $L^{p, q}(G)$ to $L^{p, r}(G)$ that commute with right translation. Then $L_{p, q}^{p, r}$ is a Banach space relative to the operator norm, and $L_{p, p}^{p, p}$ is just $L_{p}^{p}(G)$ with an equivalent norm.

As we observed in the previous section, $L_{1}^{1}(G) \subset L_{2}^{2}(G)$; therefore the two spaces form an interpolation pair. Fix parameters $\theta$ and $q$, with $0<\theta<1$, and $1 \leqslant q \leqslant \infty$. Let

$$
1 / p=(1-\theta) / 2+\theta / 1
$$

let $r$ and $s$ be indices in the interval $[1, \infty]$ satisfying the relation

$$
1 / s=1 / r+1 / q-1
$$

The following theorem generalizes a result of Zafran [Zaf 2].

THEOREM 3. Let $G$ be a locally compact group, and let the parameters $\theta, p, q, r$, and s satisfy the relations above. Then

$$
\left(L_{2}^{2}(G), L_{1}^{1}(G)\right)_{\theta, q} \subset L_{p, r}^{p, s},
$$

and the inclusion mapping is continuous.

Proof. Adopt the temporary notations $D, E$, and $F$ for the intermediate spaces $\left(L_{2}^{2}(G), L_{1}^{1}(G)\right)_{\theta, q},\left(L^{2}(G), L^{1}(G)\right)_{\theta, r}$, and $\left(L^{2}(G), L^{1}(G)\right)_{\theta, s}$. Since $D \subset L_{2}^{2}(G)$, the elements of $D$ are convolution operators with domain $C_{c}(G)$.

On the other hand, since $C_{c}(G)$ is dense in $L^{1}(G)$, we can regard $L_{1}^{1}(G)$ as a space of bounded operators on $L^{1}(G)$; similarly, we can regard $L_{2}^{2}(G)$ as a space of bounded operators on $L^{2}(G)$. Consider the bilinear map

$$
\left.S: L_{1}^{1}(G) \times\left[L^{2}(G) \cap L^{1}(G)\right)\right] \rightarrow L^{2}(G) \cap L^{1}(G),
$$

defined by letting $S(T, f)=T f$. Clearly

$$
\|S(T, f)\|_{2} \leqslant\|T\|_{L_{2}^{2}}\|f\|_{2} \text { and }\|S(T, f)\|_{1} \leqslant\|T\|_{L_{1}^{1}}\|f\|_{1},
$$

for all $T$ and $f$. It therefore follows from Zafran's multilinear interpolation theorem [Zaf 1], that there exists a constant $C$, depending only on $\theta$ and $q$, such that $\|S(T, f)\|_{F} \leqslant C\|T\|_{D}\|f\|_{E}$, for all $T$ in $L_{1}^{1}(G)$, and all $f$ in $L^{2}(G) \cap$ $L^{1}(G)$. In Zafran's statement of the theorem, the constant $C$ is equal to 1 , but he uses a different definition for the intermediate spaces, and this leads to norms 
on these spaces that are equivalent to but not equal to the norms that we use. We rephrase the inequality above as

$$
\|T f\|_{F} \leqslant C\|T\|_{D}\|f\|_{E}
$$

for all $T$ in $L_{1}^{1}(G)$ and all $f$ in $L^{2}(G) \cap L^{1}(G)$.

If $q<\infty$, then the space $L_{1}^{1}(G) \cap L_{2}^{2}(G)$, which is just $L_{1}^{1}(G)$, is dense in $D$ [BB, p. 170]; therefore inequality (19) holds for all $T$ in $D$ and all $f$ in $C_{c}(G)$. Now the space $E$ is just $L^{p, r}(G)$, with an equivalent norm, and the space $F$ is just $L^{p, s}(G)$, with an equivalent norm. Thus inequality (19) states that if $T \in D$, then $T \in L_{p, r}^{p, s}$, and that the inclusion $D \rightarrow L_{p, r}^{p, s}$ is continuous.

If $q=\infty$, then $r=1$, and $s=\infty$; now $L_{1}^{1}(G)$ is not dense in $D$, but there exists a constant $K$ such that each element of $T$ of $D$ is the limit, relative to the norm in $L_{2}^{2}(G)$, of a sequence $\left(T_{n}\right)_{n=1}^{\infty}$ of elements of $L_{1}^{1}(G)$ with $\left\|T_{n}\right\|_{D} \leqslant$ $K\|T\|_{D}$ for all $n$. Fix such an element $T$ of $D$ and such a sequence $\left(T_{n}\right)_{n=1}^{\infty}$; fix $f$ in $C_{c}(G)$. Then the sequence $\left(T_{n} f\right)_{n=1}^{\infty}$ converges, in $L^{2}(G)$, to $T f$. Hence $T_{n} f \rightarrow T f$ in measure. Now $E=L^{p, 1}(G)$ and $F=L^{p, \infty}(G)$; therefore, by inequality (19), $\left\|T_{n} f\right\|_{p, \infty} \leqslant C K\|T\|_{D}\|f\|_{p, 1}$ for all $n$. It follows that $T f \in$ $L^{p, \infty}(G)$, and that $\|T f\|_{p, \infty} \leqslant C K\|T\|_{D}\|f\|_{p, 1}$. This completes the proof of the theorem.

Inclusion (18) seems to be strict in general, because $L_{1}^{1}(G)$ is dense in $\left(L_{2}^{2}(G), L_{1}^{1}(G)\right)_{\theta, q}$, relative to the norm in $L_{2}^{2}(G)$, while, if $G$ is infinite, compact, and abelian, then $L_{1}^{1}(G)$ is not dense in $L_{p, r}^{p, s}$ relative to the norm in $L_{2}^{2}(G)$. Indeed, there exists a subset $E$ of the dual group that is a $\Lambda(q)$ set for all finite $q$, but is not a Sidon set [Ebe]; then every bounded function on $E$ can be uniformly approximated by restrictions of transforms of elements of $L_{p, r}^{p, s}$, but there exist bounded functions on $E$ that cannot be uniformly approximated by restrictions of Fourier-Stieltjes transforms [F-T, p. 31]. Sets with similar properties exist in the circle group [Sta]. Finally, the question of the density of $L_{p}^{p}(G)$ in $L_{q}^{q}(G)$, where $1<p<q \leqslant 2$, is settled in [GI].

We now come to the main result of this section.

THEOREM 4. Let $G$ be an infinite, locally compact group, and let $1<p$ $<2$. Then the inclusion $L_{p}^{p}(G) \subset L_{p, p}^{p, \infty}$ is strict.

Proof. It suffices to show that the norms on the two spaces of convolution operators are not equivalent. Denote these norms by $\|\cdot\|_{\text {strong }}$ and $\|\cdot\|_{\text {weak }}$

Construct Rudin-Shapiro measures $\left(\rho_{n}\right)_{n=0}^{\infty}$ as in the previous section, and choose disjoint left translates $\left(\rho_{n}^{\prime}\right)_{n=0}^{\infty}$ of these measures. Again, let $\mu_{N}=$ $\Sigma_{n=0}^{N} 2^{-n / p} \rho_{n}^{\prime}$. By convolving $\mu_{N}$ with a function in $C_{c}(G)$ with sufficiently small support, we see that 


$$
\begin{aligned}
\left\|\mu_{N}\right\|_{\text {strong }} & \geqslant\left(\sum_{x \in G}\left|\mu_{N}(\{x\})\right|^{p}\right)^{1 / p}=\left[\sum_{n=0}^{N}\left(2^{-n / p}\right)^{p} \cdot 2^{n}\right]^{1 / p} \\
& =(N+1)^{1 / p} .
\end{aligned}
$$

To estimate $\left\|\mu_{N}\right\|_{\text {weak }}$, we shall use Theorem 3, with $\theta$ defined by equation (16), and with $q=p^{\prime}, r=p$, and $s=\infty$. To this end, let $A_{0}=L_{2}^{2}(G)$ and $A_{1}=L_{1}^{1}(G)$, and denote the norms on those spaces by $\|\cdot\|_{0}$ and $\|\cdot\|_{1}$ respectively. By Lemma 1, we have that $\left\|\rho_{n}\right\|_{0} \leqslant \sqrt{2} 2^{n / 2}$; by Wendel's theorem, $\left\|\rho_{n}\right\|_{1}=2^{n}$. Since left translation is an isometry of $A_{0}$ and $A_{1}$, these norm estimates also hold with $\rho_{n}^{\prime}$ in place of $\rho_{n}$. We now estimate $K\left(t ; \mu_{N}\right)$.

Suppose first that $t>1$. Write $\mu_{N}=\mu_{N}+0$. Then

$$
K\left(t ; \mu_{N}\right) \leqslant\left\|\mu_{N}\right\|_{0}+t\|0\|_{1} \leqslant \sqrt{2} \sum_{n=0}^{N} 2^{n(1 / 2-1 / p)} .
$$

Since $1 / p>1 / 2$, this sum is bounded above by a constant $C$ that is independent of $N$. Hence

$$
\int_{1}^{\infty}\left[t^{-\theta} K\left(t ; \mu_{N}\right)\right]^{p^{\prime}} \frac{d t}{t} \leqslant C^{p^{\prime}} \int_{1}^{\infty} t^{-1-\theta p^{\prime}} d t .
$$

Suppose next that $t \leqslant 2^{-N / 2}$. Write $\mu_{N}=0+\mu_{N}$. Then

$$
K\left(t ; \mu_{N}\right) \leqslant\|0\|_{0}+t\left\|\mu_{N}\right\|_{1} \leqslant t \sum_{n=0}^{N} 2^{n(1-1 / p)} \leqslant D t 2^{N / p^{\prime}},
$$

for a constant $D$ that is independent of $N$. Hence

$$
\int_{0}^{2^{-N / 2}}\left[t^{-\theta} K\left(t ; \mu_{N}\right)\right]^{p^{\prime}} \frac{d t}{t} \leqslant D^{p^{\prime}} 2^{N} \int_{0}^{2^{-N / 2}} t^{(1-\theta) p^{\prime}-1} d t .
$$

Now $1-\theta=2 / p^{\prime}$, by equation (16). Therefore, the integral on the right simplifies to $\int_{0}^{2-N / 2} t d t$, and we conclude that

$$
\int_{0}^{2^{-N / 2}}\left[t^{-\theta} K\left(t ; \mu_{N}\right)\right]^{p^{\prime}} \frac{d t}{t} \leqslant \frac{D^{p^{\prime}}}{2} .
$$

Finally, suppose that $2^{-N / 2}<t \leqslant 1$. Let $M$ be the integer such that $2^{-M / 2}<t \leqslant 2^{-(M-1) / 2}$; then $0<M \leqslant N$. Write

$$
\mu_{N}=\sum_{n=M}^{N} 2^{-n / p} \rho_{n}^{\prime}+\sum_{n=0}^{M-1} 2^{-n / p} \rho_{n}^{\prime}
$$

then, much as above,

$$
K\left(t ; \mu_{N}\right) \leqslant E\left[2^{-M(1 / p-1 / 2)}+t 2^{(M-1) / p^{\prime}}\right] .
$$

for a constant $E$ that is independent of $N$. Using the fact that $2^{-M / 2}<t \leqslant$ $2^{-(M-1) / 2}$, we have that 


$$
K\left(t ; \mu_{N}\right) \leqslant E\left[t^{2(1 / p-1 / 2)}+t^{\left(1-2 / p^{\prime}\right)}\right]=2 E t^{\theta}
$$

by equation (16). Therefore,

$$
\int_{2}^{1}\left[-N / 2\left[t^{-\theta} K\left(t ; \mu_{N}\right)\right]^{p^{\prime}} \frac{d t}{t} \leqslant(2 E)^{p^{\prime}}\left(\frac{N}{2}\right) \log 2 .\right.
$$

Let $\|\cdot\|_{\text {int }}$ denote the norm in the space $\left(L_{2}^{2}(G), L_{1}^{1}(G)\right)_{\theta, p^{\prime}}$. Then inequalities (21), (22), and (23) imply that $\left\|\mu_{N}\right\|_{\text {int }}=O\left(N^{1 / p^{\prime}}\right)$ as $N \rightarrow \infty$. Therefore, by Theorem 3, $\left\|\mu_{N}\right\|_{\text {weak }}=O\left(N^{1 / p^{\prime}}\right)$ as $N \rightarrow \infty$. On the other hand, inequality (20) implies that $\left\|\mu_{N}\right\|_{\text {strong }} \neq O\left(N^{1 / p^{\prime}}\right)$ as $N \rightarrow \infty$, because $1 / p>1 / p^{\prime}$. We conclude that the norms in the spaces $L_{p}^{p}(G)$ and $L_{p, p}^{p, \infty}$ are not equivalent. This completes the proof of the theorem.

The argument used above clearly does not work if $p>2$, and it remains unknown, for such $p$, whether there exist convolution operators of weak type $(p, p)$ that are not of strong type $(p, p)$. It does follow easily from Theorem 4 , however, that, if $G$ is infinite, and $p>2$, then there exist convolution operators that are of restricted weak type $(p, p)$ but not of strong type $(p, p)$. A stronger result will be proved in Appendix 2.

COROllary 5. Let $G$ be an infinite, locally compact group, and let $1<$ $p<2$, or $2<p<\infty$. Then the inclusion $L_{p}^{p}(G) \subset L_{p, 1}^{p, \infty}$ is strict.

Proof. If $1<p<2$, then this is immediate from Theorem 4. If $p>2$, we argue as follows. The duality argument used in the proof of Lemma 1 shows that $\mu_{N}^{\vee}$, the reflection of $\mu_{N}$, has the same norm in $L_{p}^{p}(G)$ as does $\mu_{N}$ in $L_{p}^{p^{\prime}}(G)$; in particular, this norm is not $O\left(N^{1 / p}\right)$ as $N \rightarrow \infty$. Similarly, $\mu_{N}^{\vee}$ has essentially the same norm in $L_{p, 1}^{p, p}$ as does $\mu_{N}$ in $L_{p^{\prime}, p^{\prime} ;}^{p^{\prime}, \infty}$ since this norm is $O\left(N^{1 / p}\right)$ as $N \rightarrow$ $\infty$, the norms in the spaces $L_{p}^{p}(G)$ and $L_{p, 1}^{p, p}$ are not equivalent, and the inclusion $L_{p}^{p}(G) \subset L_{p, 1}^{p, p}$ is strict; a fortiori the inclusion $L_{p}^{p}(G) \subset L_{p, 1}^{p, \infty}$ is also strict. This completes the proof of the corollary.

REMARK 2. Theorem 4 was proved for various classical, locally compact, abelian groups by Zafran [Zaf 2]. For the case where $G$ is the group of integers, his proof involved the construction of a function on the circle that was smooth enough to be a multiplier of weak type $(p, p)$, but whose Fourier coefficients did not belong to $\ell^{p}$, so that the function could not be a multiplier of strong type $(p, p)$. The result for other groups then followed by mapping arguments. A similar program, starting with the case where $G$ is the weak product of countably many groups of fixed finite order, led to a proof of Theorem 4 for all infinite, locally compact, abelian groups, and all infinite, compact groups [Fou]. The method that we have used here is simpler than these methods and it yields the result in full generality. 
REMARK 3. To get an explicit example, when $1<p<2$, of a convolution operator that is of weak type $(p, p)$ but not of strong type $(p, p)$, we again consider the series

$$
T_{\delta}=\sum_{n=0}^{\infty}(n+1)^{-\delta} 2^{-n / p} \rho_{n}^{\prime},
$$

which converges in $L_{q}^{q}(G)$ whenever $|1 / q-1 / 2|<|1 / p-1 / 2|$. Now

$$
\left\|T_{\delta}\right\|_{L q} \geqslant\left\{\sum_{n=0}^{\infty}\left[(n+1)^{-\delta} 2^{-n / p}\right]^{q} \cdot 2^{n}\right\}^{1 / q}
$$

If $\delta \leqslant 1 / p$, then $\left\|T_{\delta}\right\|_{L} g$ is unbounded as $q$ approaches $p$ from above, and $T_{\delta}$ cannot be of strong type $(p, p)$. On the other hand, if $\delta>1 / p^{\prime}$, then, summing by parts, and using the estimates obtained above for $\left\|\mu_{N}\right\|_{\text {weak }}$, we can see that the series (15) converges in $L_{p, p}^{p, \infty}$. Hence $T_{\delta}$ has the desired properties whenever $1 / p^{\prime}<\delta \leqslant 1 / p$.

4. Noninclusions among spaces $L_{p}^{q}(G)$ with $q \neq p$. The inclusions that hold between spaces $L_{p}^{q}(G)$, when $q \neq p$, depend on the group $G$. For instance, if $G$ is compact, then $L_{p}^{q}(G) \subset L_{r}^{s}(G)$ whenever $p \leqslant r$ and $q \geqslant s$, because $L^{r}(G)$ $\subset L^{p}(G)$, and $L^{q}(G) \subset L^{s}(G)$ in this case; on the other hand, if $G$ is discrete, then $L_{p}^{q}(G) \subset L_{r}^{s}(G)$ whenever $p \geqslant r$ and $q \leqslant s$. In some cases, inclusion occurs because the smaller space is trivial. In fact, $L_{p}^{q}(G)=\{0\}$ if $G$ is not compact and $q<p$, or if $G$ is not unimodular and $q>p$ [BE, p. 312]; in all other cases, however, the space $L_{p}^{q}(G)$ is nontrivial.

In this section, we present noninclusion results that follow easily from the properties of Rudin-Shapiro measures and polynomials. Related results that depend on the structure of $G$ are presented in Appendix 1.

Suppose that $G$ is nondiscrete, noncompact, and unimodular. Then the Rudin-Shapiro measures $\rho_{n}$ constructed in $\S 2$, being finite sums of point masses, cannot belong to $L_{p}^{q}$ if $p<q$; to see this, compare $\left\|\rho_{n} * g\right\|_{q}$ with $\|g\|_{p}$ when $g$ is the characteristic function of a very small open set. To get convolution operations of type $(p, q)$ with $q>p$, we fatten the measures $\rho_{n}$ as follows. Fix a compact, symmetric neighbourhood $K$ of the identity with $m(K)<1$, and let $V=K^{2}$. As we observed at the end of $\S 2$, we can construct Rudin-Shapiro measures $\left(\rho_{n}\right)_{n=0}^{\infty}$ so that the sets $y_{k} V$ are disjoint, as are the sets $V y_{k}$. Denote the support of $\rho_{n}$ by $S_{n}$, and choose an element $z_{n}$ of $G$ so that $z_{n} \notin K^{3} S_{n} K^{3} S_{n}^{-1}$. Now let $f$ be a nonnegative, symmetric, continuous function supported by $K$, with $\|f\|_{1}=\|f\|_{\infty}=f(e)=1$. Finally, let

$$
F_{n}=L\left(z_{n}\right) \rho_{n} * f+f * \rho_{n} .
$$

Then the map $g \mapsto F_{n} * g$ defines an element of $L_{p}^{q}(G)$ whenever $1 \leqslant p \leqslant q \leqslant \infty$. 
Indeed, by Young's inequality for convolution, $f \in L_{p}^{q}(G)$ for all such $p$ and $q$, while $\rho_{n} \in L_{p}^{p}(G) \cap L_{q}^{q}(G)$.

Suppose next that $G$ is discrete and infinite, with its Haar measure normalized so that $m(\{e\})=1$. Then the construction of $F_{n}$ given above can be repeated in this case; in fact, matters are simpler, because $f$ must be the characteristic function of $\{e\}$, and formula (24) simplifies to $F_{n}=L\left(z_{n}\right) \rho_{n}+\rho_{n}$.

We now generalize the main estimates obtained in Lemma 1.

LEMMA 6. Let $1 \leqslant p \leqslant q \leqslant \infty$, and let $t=\min \left(q, p^{\prime}\right)$. Then there exists a positive constant $C_{p, q}$ such that

$$
C_{p, q} 2^{n / t} \leqslant\left\|F_{n}\right\|_{L_{p}^{q}} \leqslant 2 \sqrt{2} 2^{n / t}
$$

for all $n$.

Proof. We deal first with the upper bound on $\left\|F_{n}\right\|_{L_{p}^{q}}$. By Lemma 1 and the fact that $\|f\|_{1}=1$, we have that

$$
\left\|F_{n}\right\|_{L_{p}^{p}} \leqslant 2\left\|\rho_{n}\right\|_{L_{p}^{p}}\|f\|_{1} \leqslant 2 \sqrt{2} 2^{n \max \left(1 / p, 1 / p^{\prime}\right)} .
$$

This is the desired upper bound when $p=q$.

Because the set $y_{k} V$ are disjoint, the function $\rho_{n} * f$ is a sum of $2^{n}$ disjointly-supported translates of $f$, and therefore, $\left\|\rho_{n} * f\right\|_{\infty}=1$. Similarly, $\left\|f * \rho_{n}\right\|_{\infty}=1$. The choice of $z_{n}$ guarantees that $L\left(z_{n}\right) \rho_{n} * f$ and $f * \rho_{n}$ are disjointly supported, so that $\left\|F_{n}\right\|_{\infty}=1$. Since $\left\|F_{n}\right\|_{L_{1}^{\infty}}=\left\|F_{n}\right\|_{\infty}$, the second inequality in (25) holds when $p=1$ and $q=\infty$.

If $p \neq q$, and $(p, q) \neq(1, \infty)$, then the point $(1 / p, 1 / q)$ lies on the line segment joining the point $(1,0)$ to some point $(1 / r, 1 / r)$ on the diagonal of the square $[0,1] \times[0,1]$. We can write

$$
(1 / p, 1 / q)=(1-\theta)(1,0)+\theta(1 / r, 1 / r)
$$

for a number $\theta$ between 0 and 1. Then, by Riesz convexity,

$$
\left\|F_{n}\right\|_{L}{ }_{p} \leqslant\left\|F_{n}\right\|_{L_{1}^{\infty}}^{(1-\theta)}\left\|F_{n}\right\|_{L_{r}^{r}}^{\theta} \leqslant 2 \sqrt{2} 2^{n \theta \max \left(1 / r, 1 / r^{\prime}\right)},
$$

by what we have already proved. But, $\theta \max \left(1 / r, 1 / r^{\prime}\right)=\max \left(1 / q, 1 / p^{\prime}\right)=1 / t$, and the proof of the second inequality in (25) is complete.

We now consider the lower bound on $\left\|F_{n}\right\|_{L_{p} q}$. Suppose first that $1 / p+$ $1 / q \geqslant 1$. Then $t=q$. The choice of $z_{n}$ guarantees that $L\left(z_{n}\right) \rho_{n} * f * f$ and $f * \rho_{n} * f$ have disjoint supports. Thus

$$
\left\|F_{n} * f\right\|_{q} \geqslant\left\|L\left(z_{n}\right) \rho_{n} * f * f\right\|_{q}=\left\|\rho_{n} * f * f\right\|_{q},
$$

On the other hand, since the sets $y_{k} V$ are disjoint, $\rho_{n} * f * f$ is a sum of $2^{n}$ 
disjointly-supported translates of $f * f$. Therefore

and

$$
\left\|\rho_{n} * f * f\right\|_{q} \geqslant 2^{n / q}\|f * f\|_{q},
$$

$$
\left\|F_{n}\right\|_{L_{p}^{q}} \geqslant\left\|F_{n} * f\right\|_{q} /\|f\|_{p} \geqslant 2^{n / q}\|f * f\|_{q} /\|f\|_{p} .
$$

This is the desired lower bound with $C_{p, q}=\|f * f\|_{q} /\|f\|_{p}$.

If $1 / p+1 / q<1$, then $t=p^{\prime}$. The adjoint of the map $g \mapsto F_{n} * g$, is the map $g \mapsto F_{n}^{\vee} * g$, where $F_{n}^{\vee}=R\left(z_{n}^{-1}\right)\left(f * \rho_{n}^{\bigvee}\right)+\rho_{n}^{\vee} * f$. An argument similar to the one in the previous paragraph shows that

$$
\left\|F_{n}^{V}\right\|_{L_{q^{\prime}}^{\prime}}^{p^{\prime}} \geqslant C_{q^{\prime}, p^{\prime 2}}{ }^{n / p^{\prime}}
$$

Since $\left\|F_{n}\right\|_{L q}=\left\|F_{n}\right\|_{L p^{\prime}}$, we get the desired lower bound with $C_{p, q}=C_{q^{\prime}, p^{\prime}}$. This completes the proof of the lemma.

Now we generalize Theorem 2.

THEOREM 7. Let $G$ be a noncompact, unimodular, locally compact group. Let $1 \leqslant p, q, r, s \leqslant \infty$. Suppose that $p \leqslant q$, and that $\min \left(s, r^{\prime}\right)<\min \left(q, p^{\prime}\right)$. Then $L_{p}^{q}(G)$ is not included in $L_{r}^{s}(G)$.

Proof. Suppose that $L_{p}^{q}(G) \subset L_{r}^{s}(G)$. Then the space $L_{r}^{s}(G)$ is nontrivial, because $F_{n} \in L_{p}^{q}(G)$; therefore $r \leqslant s$. By the closed-graph theorem, the injection $L_{p}^{q}(G) \rightarrow L_{r}^{s}(G)$ must be continuous. The estimates given in Lemma 6 for $\left\|F_{n}\right\|_{L} g$ and $\left\|F_{n}\right\|_{L_{r}^{s}}$ imply that such a continuous injection can exist only if $\min \left(s, r^{\prime}\right) \geqslant \min \left(q, p^{\prime}\right)$. Hence $L_{p}^{q}(G) \not \subset L_{r}^{s}(G)$ if $\min \left(s, r^{\prime}\right)<\min \left(q, p^{\prime}\right)$, and the proof of the theorem is complete.

We now consider inclusions among the spaces $L_{p}^{q}(G)$ when $q \geqslant p^{\prime}$, and $G$ is compact. Our next theorem improves slightly a result of Figa-Talamanca and Price [F-T P 1], [F-TP 2].

THEOREM 8. Let $G$ be an infinite, compact group. Let $1 \leqslant p, q, r, s \leqslant$ $\infty$. Suppose that $p \leqslant q$, and that $\min \left(r, s^{\prime}\right)<\min \left(p, q^{\prime}\right)$. Then $L_{p}^{q}(G)$ is not included in $L_{r}^{s}(G)$.

Proof. Figà-Talamanca and Price prove this except in the case where $q=p^{\prime}$. To extend their proof to this case, we need only show that if $0<\alpha<$ $2 / p^{\prime} \leqslant 2 / p$, then there exists a finite index $s$ such that

$$
\|f\|_{L_{p}^{p^{\prime}}} \leqslant\|f\|_{L_{2}^{2}}^{\alpha}\|f\|_{s}^{1-\alpha},
$$

for all trigonometric polynomials $f$. Since the point $\left(1 / p, 1 / p^{\prime}\right)$ lies on the line segment joining $(1,0)$ to $(1 / 2,1 / 2)$, there exists an index $t$ such that $\left(1 / t, 1 / t^{\prime}\right)$ 
lies strictly between $\left(1 / p, 1 / p^{\prime}\right)$ and $(1,0)$ on this line segment, and such that

$$
\left(1 / p, 1 / p^{\prime}\right)=(1-\alpha)\left(1 / t, 1 / t^{\prime}\right)+\alpha(1 / 2,1 / 2)
$$

By Riesz convexity,

$$
\|f\|_{L_{p}}^{p^{\prime}} \leqslant\|f\|_{L_{2}^{2}}^{\alpha}\|f\|_{L_{t}^{t^{\prime}}}^{(1-\alpha)}
$$

Now let $s=t^{\prime} / 2$. Then, by Young's inequality for convolution, $\|f\|_{L_{t}} \leqslant\|f\|_{s}$, and inequality (26) is proved. The rest of the proof of the theorem proceeds as in [F-TP 1] and [F-TP 2], and we omit it.

We continue to suppose that $G$ is compact. If $1 \leqslant q \leqslant 2 \leqslant p$, then $L_{p}^{q}(G)$ $=L_{2}^{2}(G)$, with equivalence of norms. Except for the case where $p=\infty$ and $q=1$, this is proved by randomization arguments in [F-TP 1] and [F-TP 2]; a different approach, due to Gilbert [Gil], gives the result in general. We remark that the case where $p=\infty$ and $q=1$ can be handled by randomization arguments. Indeed, by the Riesz-Thorin theorem, if $1<q<2$, then $\|g\|_{L} \leqslant \leqslant\|g\|_{L \infty}^{\theta}\|g\|_{L_{\infty}^{2}}^{1-\theta}$ for the appropriate $\theta$ in $(0,1)$ and arbitrary continuous $g$. Since $L_{\infty}^{2}(G)$ and $L_{\infty}^{q}(G)$ have norms equivalent to $L_{2}^{2}(G),\|g\|_{L_{\infty}^{1}} \geqslant C\|g\|_{L_{2}^{2}}$. The inequality $\|g\|_{L_{2}^{2}} \geqslant C^{\prime}\|g\|_{L_{\infty}^{1}}$ being evident, and continuous functions being dense in $L_{\infty}^{1}(G)$ in the strong operator topology, the remark is verified.

On the other hand, if $1 \leqslant q<p<2$, then $L_{p}^{q}(G)=L_{p, p}^{p, \infty}$, again with equivalence of norms [Saw]. Finally, a duality argument shows that, if $2<q<$ $p \leqslant \infty$, then $L_{p}^{q}(G)=L_{q, 1}^{q, q}$, with equivalence of norms. Interpolation arguments show that if $1<p<2$, then $L_{r}^{r}(G) \subset L_{p, p}^{p, \infty} \subset L_{s}^{s}(G)$ whenever $1 \leqslant r \leqslant p<s \leqslant$ 2; similarly, if $2<q<\infty$, then $L_{r}^{r}(G) \subset L_{q, 1}^{q, q} \subset L_{s}^{s}(G)$ whenever $2 \leqslant s<q \leqslant r$.

We conclude that, in Theorem 8 , the assumption that $p \leqslant q$ can be replaced by the weaker assumption that $\min \left(r, s^{\prime}\right)<2$; the latter assumption is essential, because, if $\min \left(r, s^{\prime}\right) \geqslant 2$, then $s \leqslant 2 \leqslant r$, and $L_{p}^{q}(G) \subset L_{r}^{s}(G)$ for all $p$ and $q$.

REMARK 4. Suppose that $G$ is noncompact but unimodular, that $1 \leqslant p<$ $q<\infty$, and that $1 / p+1 / q>1$; then there exists a convolution operator on $G$ that is of weak type $(p, q)$ but not of strong type $(p, q)$. We sketch the proof. First, write

$$
(1 / p, 1 / q)=(1-\theta)(1,0)+\theta(1 / r, 1 / r),
$$

for suitable indices $r$ and $\theta$. Then, much as in the proof of Theorem 3, we have that $\left(L_{1}^{\infty}(G), L_{r}^{r}(G)\right)_{\theta, p^{\prime}} \subset L_{p, p}^{q, \infty}$, with continuous inclusion. Choose left translates $F_{n}^{\prime}$ of the functions $F_{n}$ considered in Lemma 6 , so that the functions $F_{n}^{\prime} * f$ have disjoint supports, and let $H_{N}=\Sigma_{n=0}^{N} 2^{-n / q} F_{n}^{\prime}$. Then the norm of $H_{N}$ in the intermediate space $\left(L_{1}^{\infty}(G), L_{r}^{r}(G)\right)_{\theta, p^{\prime}}$ is $O\left(N^{1 / p^{\prime}}\right)$ as $N \rightarrow \infty$, while the norm of $H_{N}$ in $L_{p}^{q}(G)$ is at least $(N+1)^{1 / q}\|f * f\|_{q} /\|f\|_{p}$. These estimates, combined with the closed-graph theorem show that $L_{p, p}^{q, \infty} \not \subset L_{p}^{q}(G)$. We do not 
know whether this noninclusion must also occur when $1 / p+1 / q \leqslant 1$, but we can show in this case that $L_{p, 1}^{q, \infty} \not \subset L_{p}^{q}(G)$.

5. Operators that commute with left translations. All our results about noninclusions of the spaces $L_{p}^{q}(G)$ also hold for the corresponding spaces $L_{p}^{q}(G)$ of operators that commute with left translations, provided that the group $G$ is unimodular. Indeed, left and right translation coincide if $G$ is abelian. If $G$ is unimodular, but not abelian, denote by $G^{\vee}$ the group that coincides with $G$ as a topological space but has the opposite multiplication; i.e. the product of $x$ and $y$ in $G$ is $y x$. Since $G$ is unimodular, its Haar measure is also a Haar measure on $G^{v}$, and $L^{p}(G)=L^{p}\left(G^{v}\right)$ for all $p$. It follows that an operator belongs to $L_{p}^{q}(G)$ if and only if it belongs to $L_{p}^{q}(G)$, and thus the inclusions and noninclusions holding for the spaces $L_{p}^{q}(G)$ also hold for the spaces $L_{p}^{q}(G)$.

Even if $G$ is not unimodular, the results in $\S \S 2$ and 3 concerning the spaces $L_{p}^{p}(G)$ and $L_{p, q}^{p, r}$ also hold for the corresponding spaces of operators that commute with left translations: the proofs of these results can be modified to work for the spaces $L_{p}^{p}(G)$ and $L_{p, q}^{p, r}$. For instance, in defining Rudin-Shapiro measures, we now set $\rho_{n+1}=\rho_{n}+R\left(x_{n}\right) \sigma_{n}$, with each $x_{n}$ chosen so that, inter alia, they belong to the (noncompact) kernel of the modular function; in the analogues of Lemma 1 and Theorems 2 and 4 , we identify $\rho_{n}$ with the operator $f \mapsto f * \rho_{n}$.

We now consider the spaces $L_{p}^{q}(G)$ for nonunimodular $G$. The proof that the spaces $L_{p}^{q}(G)$ are trivial if $q<p$ also works for $L_{p}^{q}(G)$; hence we assume that $q \geqslant p$. If $g \in C_{c}(G)$, the map $f \mapsto f * g$ defines an element of $L_{p}^{q}(G)$ whenever $1 \leqslant p \leqslant q \leqslant \infty$; we identify $g$ with this operator. We follow Hewitt and Ross [HR, §20] in our definition of the modular function $\Delta$.

THEOREM 9. Let $G$ be a nonunimodular locally compact group; suppose $1 \leqslant p \leqslant q \leqslant \infty$, and $g \in C_{c}(G)$. Then, for all $x$ in $G$,

$$
\|L(x) g\|_{L_{p}^{q}}=\Delta(x)^{-1 / p}\|g\|_{L_{p}^{q}}
$$

and

$$
\|R(x) g\|_{L_{p}^{q}}=\Delta(x)^{-1 / q^{\prime}}\|g\|_{L_{p}^{q}}
$$

Proof. Observe that $f * R(x) g=R(x)(f * g)$, for all $f$ in $C_{c}(G)$, so that

$$
\|f * R(x) g\|_{q}=\Delta(x)^{-1 / q^{\prime}}\|f * g\|_{q} \leqslant \Delta(x)^{-1 / q^{\prime}}\|f\|_{p}\|g\|_{L_{p}^{q}},
$$

and thus

$$
\|R(x) g\|_{L_{p}^{q}} \leqslant \Delta(x)^{-1 / q^{\prime}}\|g\|_{L_{p}^{q}}
$$

Since $g=R\left(x^{-1}\right) R(x) g$, we have also that 


$$
\|g\|_{L_{p}^{q}} \leqslant \Delta\left(x^{-1}\right)^{-1 / q^{\prime}}\|R(x) g\|_{L_{p}^{q}}
$$

and the equality (28) is proved.

$$
\begin{gathered}
\text { Next, for arbitrary } f \text { in } C_{c}(G), f * L(x) g=[R(x) f] * g \text { so that } \\
\|f *[L(x) g]\|_{q} \leqslant\|[R(x) f] * g\|_{q} \leqslant\|R(x) f\|_{p}\|g\|_{L_{p}^{q}}=\Delta(x)^{-1 / p^{\prime}}\|f\|_{p}\|g\|_{L_{p}^{q}},
\end{gathered}
$$

and

$$
\|L(x) g\|_{L q} \leqslant \Delta(x)^{-1 / p^{\prime}}\|g\|_{L_{p}^{q}}
$$

The equality (27) then follows from the identity $g=L\left(x^{-1}\right) L(x) g$.

COROLlaRY 10. Let $G$ be a nonunimodular locally compact group. Then no inclusions hold between distinct spaces $L_{p}^{q}(G)(1 \leqslant p \leqslant q \leqslant \infty)$.

Proof. Since $\Delta(x)$ can be made arbitrarily large or small, the equalities (27) and (28) imply that no inclusions between distinct spaces $L_{p}^{q}(G)$ can be continuous. On the other hand, the closed graph theorem implies that any such inclusion must be continuous. Hence there are no inclusions between distinct spaces $L_{p}^{q}(G)$.

The preceding corollary suggests we should study rather different inclusions if $G$ is not unimodular. Given an operator $T$ from $C_{c}(G)$ to $L_{\text {loc }}^{1}(G)$, we define $\left(\Delta^{\theta} T\right)$, for any complex number $\theta$ thus: $\left(\Delta^{\theta} T\right) f=\Delta^{\theta} \cdot\left[T\left(\Delta^{-\theta} \cdot f\right)\right], \Delta^{-\theta} \cdot f$ and $\Delta^{\theta} \cdot\left[T\left(\Delta^{-\theta} f\right)\right]$ being the usual pointwise products. Note that if, for some $g$ in $L_{\text {loc }}^{\mathbf{1}}(G), T f=f * g$ then $\left(\Delta^{\theta} T\right) f=f *\left(\Delta^{\theta} \cdot g\right)$, where, again, $\Delta^{\theta} \cdot g$ is the pointwise product. We define $\Delta^{\theta} L_{p}^{q}(G)$ in the obvious manner.

LEMMA 11. Let $G$ be a nonunimodular locally compact group; suppose $1 \leqslant p, q, s \leqslant \infty$, and $1 / p-1 / q=1-1 / s$. Let $\theta=1 / p^{\prime}$. Then $\Delta^{\theta} L_{1}^{s}(G) \subset$ $L_{p}^{q}(G)$.

Proof. We use the complex interpolation theorem of Stein [Ste 1], which states that if $\left\{T_{z}: z \in C: \operatorname{Re}(z) \in[0,1]\right\}$ is an analytic family of operators such that $\left\|T_{j+i y}(f)\right\|_{q_{j}} \leqslant M_{j}\|f\|_{p_{j}}$ whenever $f \in C_{c}(G), y \in R$, and $j=0,1$, then $\left\|T_{\theta} f\right\|_{q} \leqslant M_{0}^{1-\theta} M_{1}^{\theta}\|f\|_{p}$ for $f$ in $C_{c}(G)$; here $0<\theta<1,1 / p=(1-\theta) / p_{0}+$ $\theta / p_{1}$ and $1 / q=(1-\theta) / q_{0}+\theta / q_{1}$.

Let us treat the case where $p=q$, so that $s=1$. Now $L_{1}^{1}(G)$ and $L_{\infty}^{\infty}(G)$ are exactly $M(G)$ and $\Delta \cdot M(G)$ respectively. If $\mu$ is a compactly supported measure, the family $\left\{\Delta^{z} \cdot \mu: z \in C, \operatorname{Re}(z) \in[0,1]\right\}$ is analytic in the interior of the strip $\{z \in C: \operatorname{Re}(z) \in[0,1]\}$ in the strong operator topology $\left(C_{0}(G)\right.$ to $C_{0}(G)$ ); using the obvious estimates, we find that $\left\|\Delta^{\theta} \cdot \mu\right\|_{L p} \leqslant\|\mu\|_{L \mathcal{H}}$. Evidently we can conclude that $\Delta^{\theta} L_{1}^{1}(G) \subset L_{p}^{p}(G)$.

The cases where $p \neq q$ are treated similarly: one interpolates between the 
known inclusions $L_{1}^{s}(G) \subset L_{1}^{s}(G)$ and $\Delta^{1 / s} L_{1}^{s}(G) \subset L_{s^{\prime}}^{\infty}(G)$.

We have shown that there are nontrivial inclusions for the spaces $\Delta^{\theta} L_{p}^{q}(G)$. The next theorem shows these inclusions are relatively simple.

THEOREM 12. Let $G$ be a nonunimodular locally compact group. If $1 \leqslant p \leqslant q \leqslant \infty, 1 \leqslant r \leqslant s \leqslant \infty$, and $L_{p}^{q}(G) \subset \Delta^{\theta} \cdot L_{r}^{s}(G)$, for some real number $\theta$, then $1 / p-1 / q=1 / r-1 / s$, and $\theta=1 / s-1 / q$.

Proof. The translation estimates of Theorem 9 hold the key to this theorem also. The inclusion hypothesized must be continuous, by the closed graph theorem; so, for a constant $C$ independent of $g$ in $C_{c}(G)$ and of $x$ in $G$,

and

$$
\begin{gathered}
\left\|\Delta^{-\theta} \cdot g\right\|_{L_{r}^{s}} \leqslant C\|g\|_{L_{p}^{q}} \\
\left\|\Delta^{-\theta} \cdot R(x) g\right\|_{L_{r}^{s}} \leqslant C\|R(x) g\|_{L_{p}^{q}},
\end{gathered}
$$

$$
\left\|\Delta^{-\theta} \cdot L(x) g\right\|_{L_{r}^{s}} \leqslant C\|L(x) g\|_{L_{p}^{q}}
$$

Now $\Delta^{-\theta} \cdot R(x) g=\Delta^{-\theta}(x) \cdot R(x)\left[\Delta^{-\theta} \cdot g\right]$; so the right translation equality (28) can be applied to inequality (30) to obtain

$$
\Delta^{-\theta}(x) \cdot \Delta(x)^{-1 / s^{\prime}}\left\|\Delta^{-\theta} g\right\|_{L_{r}^{s}} \leqslant \Delta(x)^{-1 / q^{\prime}\|g\|_{L q} \cdot}
$$

In order that this inequality be compatible with inequality (29), it is necessary (and sufficient) that $\theta=1 / s-1 / q$. Applying the left translation equality in a similar way, we show also that $\theta=1 / r-1 / p$, whence the theorem.

Our final result for nonunimodular groups is proved using fattened RudinShapiro measures, much as in Theorem 7. We omit the proof.

THEOREM 13. Let $G$ be a nonunimodular locally compact group. Suppose that $1 \leqslant p \leqslant q \leqslant \infty$, and $1 \leqslant r \leqslant s \leqslant \infty$, that $\theta$ is real, and $L_{p}^{q}(G) \subset \Delta^{\theta} L_{r}^{s}(G)$. Then $\min \left(s, r^{\prime}\right) \geqslant \min \left(q, p^{\prime}\right)$.

Appendix 1. Group structure and noninclusions for the spaces $L_{p}^{q}(G)$. In this appendix, we sketch some further relations between structure of the group $G$ and inclusions for the spaces $L_{p}^{q}(G)$, generalising results of R. Lipsman [Lip 4] and M. Rajagopolan [Raj 1], [Raj 2]. In view of the results of the previous section, we restrict our attention to unimodular groups. Proofs of the next three theorems are given below.

THEOREM 14. Let $G$ be a unimodular nondiscrete locally compact group; suppose $1 \leqslant p, q, r, s \leqslant \infty$, and $0 \leqslant 1 / p-1 / q<1 / r-1 / s$. Then $L_{p}^{q}(G)$ is not included $L_{r}^{s}(G)$. 
REMARK 5. For infinite compact abelian groups, this theorem and Theorem 8 combine to give best possible results. For, if $G$ is abelian, $L_{p}^{q}(G)=$ $L_{q}^{p^{\prime}}(G)$; applying the Riesz-Thorin theorem, and using the fact that $L_{p}^{q}(G) \subset$ $L_{r}^{s}(G)$ whenever $p \leqslant r$ and $q \geqslant s$, we find that $L_{p}^{q}(G)$ is contained in all the spaces $L_{r}^{s}(G)$ not specifically excluded by Theorems 8 and 14 . For some nonabelian

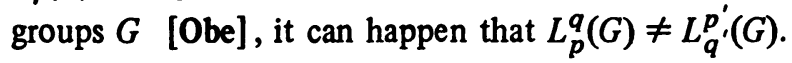

Before stating the next theorem, we remind the reader that a locally compact group $G$ is said to be near-connected if it is compact modulo the connected component of the identity, that the radical of a Lie group is the unique maximal connected solvable normal subgroup, and that a Lie group is said to be semisimple if its radical is trivial.

THEOREM 15. Let $G$ be a unimodular, locally compact group; $1 \leqslant p, q, r$, $s \leqslant \infty$, and $1 / p-1 / q>1 / r-1 / s \geqslant 0$. If $L_{p}^{q}(G)$ is included in $L_{r}^{s}(G)$, then $G$ has an open near-connected subgroup $G_{1}$, which has a compact normal (in $G_{1}$ ) subgroup $N$ such that $G_{1} / N$ is a semisimple Lie group with finite centre.

THEOREM 16. Let $G$ be a unimodular, locally compact group; suppose that $1 \leqslant p, q, r, s \leqslant \infty$, that $1 / p-1 / q>1 / r-1 / s \geqslant 0$, and that $L_{p}^{q}(G) \subset L_{r}^{s}(G)$. Then every compact open subgroup of $G$ is contained in a maximal compact open subgroup of $G$ of uniformly bounded Haar measure; for any compact open subgroup $K$ of $G$ and any $x$ in $G$, either $x^{n} \in K$ for some integer $n$, or no relation $x^{n} K=K x^{m}$, with $m, n, \neq 0$, can hold.

REMARK 6. If an infinite discrete group satisfies the hypotheses of Theorem 16, it must contain a Burnside group, i.e. a finitely generated infinite group in which every element is of bounded order. To see this, take $K=\{e\}$ in the theorem above.

REMARK 7. We are unable to combine Theorems 15 and 16 to give restrictions on both the identity component $G_{0}$ and the quotient group $G / G_{0}$ for a group $G$ satisfying the hypotheses of Theorem 15 . It seems plausible that such a combination might be made.

The proofs of Theorems 14 and 15 depend on Yamabe's structure theorem, which states that any locally compact group $G$ has an open near-connected subgroup $G_{1}$, which has a compact normal subgroup $N$ such that $G_{1} / N$ is a connected Lie group. D. Montgomery and L. Zippin [MZ, 2.6, 2.7, 4.6] give full details of this result. The proof of Theorem 14 hinges on the following lemma.

LEMMA 17. Suppose $G$ is a nondiscrete locally compact group. There exist a sequence of relatively compact neighbourhoods $\left(U_{n}\right)$ of the identity in $G$ such that 


$$
m\left(U_{n}^{2}\right) \leqslant C m\left(U_{n}\right), \quad n=1,2, \ldots ; \quad m\left(U_{n}\right) \rightarrow 0 \quad \text { as } n \rightarrow \infty
$$

where $C$ is a constant independent of $n$, and $m\left(U_{n}\right)$ is the Haar measure of the set $U_{n}$.

Proof. If $G$ is totally disconnected, then the family of compact open subgroups of $C$ is a basis for the topology of $G$ at the identity. Take $\left(U_{n}\right)$ to be any (strictly) decreasing sequence of such subgroups. Clearly this $\left(U_{n}\right)$ satisfies the required conditions.

If the identity component $G_{0}$ of $G$ is compact but not open, we take a similar sequence in the quotient $G / G_{0}$ and carry it back to $G$ by means of the canonical projection of $G$ onto $G / G_{0}$.

If the identity component $G_{0}$ of $G$ is compact and open, we can find a normal subgroup $N$ of $G_{0}$ such that $G_{0} / N$ is a compact Lie group (see [FTP 2]). Such a sequence $\left(U_{n}\right)$ of neighbourhoods is known to exist in a Lie group [EH]; it can be carried back to $G_{0}$ by means of the canonical projection.

Finally, if the identity component $G_{0}$ of $G$ is not compact, we apply Yamabe's theorem: $G$ has an open, near-connected subgroup $G$, with a compact normal subgroup $N$ such that $G_{1} / N$ is a Lie group. A sequence can be found in $G_{1} / N$ and carried back to $G_{1}$.

Proof of Theorem 14. We estimate the $L_{p}^{q}(G)$ and $L_{r}^{s}(G)$ norms of the characteristic function $\chi_{U_{n}}$ of $U_{n}$, where $U_{n}$ is a member of a sequence of neighbourhoods as in Lemma 17.

Let $t$ be the number such that $1-1 / t=1 / p-1 / q$. Then $L^{t}(G)$ is embedded continuously in $L_{p}^{q}(G)$, and

$$
\left\|\chi_{U_{n}}\right\|_{L_{p}^{q}} \leqslant\left\|\chi_{U_{n}}\right\|_{t}=m\left(U_{n}\right)^{1-1 / p+1 / q} .
$$

Now observe that $m\left(U_{n}\right) \chi_{U_{n}^{-1}} \leqslant \chi_{U_{n}} * \chi_{U_{n}^{-2}}$ so

$$
m\left(U_{n}\right)\left\|\chi_{U_{n}^{-1}}\right\|_{s} \leqslant\left\|\chi_{U_{n}}\right\|_{L_{r}^{s}}\left\|\chi_{U_{n}^{-2}}\right\|_{r},
$$

i.e. $m\left(U_{n}\right) m\left(U_{n}\right)^{1 / s} \leqslant\left\|\chi_{U_{n}}\right\|_{L_{r}^{s}} m\left(U_{n}^{2}\right)^{1 / r}$. With the constant $C$ of Lemma 17, it follows that

$$
\left\|\chi_{U_{n}}\right\|_{L_{r}^{s}} \geqslant C^{-1 / r} m\left(U_{n}\right)^{1-1 / r+1 / s}
$$

These two estimates, together with the fact that $m\left(U_{n}\right)$ can be arbitrarily small, imply that $L_{p}^{q}(G)$ cannot be imbedded continuously in $L_{r}^{s}(G)$; hence, by the closed graph theorem, $L_{p}^{q}(G)$ is not contained in $L_{r}^{s}(G)$.

The proof of Theorem 15 is rather long, and will be presented after a series of five lemmas. The first of these is motivated by Yamabe's theorem.

LEMMA 18. Suppose $G_{1}$ is an open subgroup of the locally compact 
group $G$, and that $N$ is a compact normal subgroup of $G_{1}$. Then, with a natural definition of support and constancy on cosets, $L_{p}^{q}\left(G_{1}\right)$ can be identified with the subspace of $L_{p}^{q}(G)$ of operators supported in $G_{1}$, and $L_{p}^{q}\left(G_{1} / N\right)$ can be identified with the subspace of $L_{p}^{q}\left(G_{1}\right)$ of operators constant on cosets of $N$ in $G_{1}$.

Proof. Say that an operator $T$ is supported in $G_{1}$ if $T(f)$ is supported in $G_{1}$ whenever $f$ is, and use the identification of $L^{p}\left(G_{1}\right)$ with the subspace of $L^{p}(G)$ of functions supported in $G_{1}$.

Write $\mu$ for the central idempotent measure (on $G_{1}$ ) carried by $N$ : for any $f$ in $C_{c}\left(G_{1}\right), \mu * f(x)=\int_{N} f(x n) d n$ and say that $T$ is constant on cosets of $N$ in $G_{1}$ if and only if $T(f)=T(\mu * f)$ for every $f$ in $C_{c}\left(G_{1}\right)$. Use the isomorphism of $L^{p}\left(G_{1} / N\right)$ with the subspace of $L^{p}\left(G_{1}\right)$ of functions constant on cosets of $N$ in $G_{1}$.

LEMMA 19. Suppose that $G$ is a unimodular locally compact group, that $1 \leqslant p, q, r, s \leqslant \infty$, that $1 / p-1 / q>1 / r-1 / s \geqslant 0$, and that $L_{p}^{q}(G) \subset L_{r}^{s}(G)$. Then $L^{t}(G) \subset L_{r}^{s}(G)$, where $1-1 / t=1 / p-1 / q$.

Proof. $L^{t}(G)$ is a subspace of $L_{p}^{q}(G)$.

LEMMA 20. Suppose that $G$ is a unimodular locally compact group, that $1 \leqslant p, q, r, s \leqslant \infty$, that $1 / p-1 / q>1 / r-1 / s \geqslant 0$, and that $L_{p}^{q}(G) \subset L_{r}^{s}(G)$. Then the centre of $G$ is compact.

Proof. Suppose the centre is noncompact. We shall reach a contradiction. Using structure theory for LCA groups [HR, 24.30], we see that $G$ contains an infinite discrete central subgroup $D$ or a noncompact central subgroup $D^{\prime}$ with a compact open subgroup $K$. In the latter case, $G / K$ has an infinite discrete central subgroup $D^{\prime} / K$; by Lemma 18 , there is no loss of generality in assuming that $G$ contains an infinite discrete central subgroup $D$.

We claim that $L^{t}(D) \subset L_{r}^{s}(D)$, where $1-1 / t=1 / p-1 / q$. To see this, take a small relatively compact neighbourhood $U$ of the identity in $G$ such that $U^{3} \cap$ $D=\{e\}$. If $f \in L^{t}(D)$, then $f^{\prime}$ such that $f^{\prime}=\Sigma_{d \in D} f(d) L(d) \chi_{U}$ belongs to $L^{t}(G)$, hence to $L_{r}^{s}(G)$, by Lemma 19 and the hypothesis that $L_{p}^{q}(G) \subset L_{r}^{s}(G)$. For $g$ and $h$ in $C_{c}(D)$, we define $g^{\prime}$ and $h^{\prime}$ analogously, and note that

$$
\begin{aligned}
\left|\left(f^{\prime} * \chi_{U} * \chi_{U}\right)\right|_{D} * g * h(e) \mid & =\left|f^{\prime} * g^{\prime} * h^{\prime}(e)\right| \\
& \leqslant\left\|f^{\prime}\right\|_{L_{r}^{s}}\left\|g^{\prime}\right\|_{r}\left\|h^{\prime}\right\|_{s^{\prime}}=C\left\|f^{\prime}\right\|_{L_{r}^{s}}\|g\|_{r}\|h\|_{s^{\prime}}
\end{aligned}
$$

for some constant $C$. The converse of Hölder's inequality implies that

$$
\left\|\left.\left(f^{\prime} * \chi_{U} * \chi_{U}\right)\right|_{D} * g\right\|_{s} \leqslant C\left\|f^{\prime}\right\|_{L_{r}^{s}}\|g\|_{r},
$$

i.e. $\left.\left(f^{\prime} * \chi_{U} * \chi_{U}\right)\right|_{D} \in L_{r}^{S}(D)$. Our choice of $U$ guarantees that $\left.\left(f^{\prime} * \chi_{U} * \chi_{U}\right)\right|_{D}$ 
$=C^{\prime} f$, for some constant $C^{\prime}$; so we conclude that $L^{t}(D) \subset L_{r}^{s}(D)$, as claimed.

Now an infinite abelian group contains a copy of the integers $Z$ or an increasing sequence of finite subgroups $D_{n}$. If $Z \subset D$, we have a contradiction:

$$
\left\|\chi_{[-N, N]} * \chi_{[-N, N]}\right\|_{s} \leqslant\left\|\chi_{[-N, N]}\right\|_{L_{r}^{s}}\left\|\chi_{[-N, N]}\right\|_{r},
$$

whence $\left\|\chi_{[-N, N]}\right\|_{L_{r}^{s}} \geqslant C^{\prime \prime} N^{1+1 / s-1 / r}$ whereas $\left\|\chi_{[-N, N]}\right\|_{t}=(2 N+1)^{1 / t}$, which the closed graph theorem shows is impossible. However, if $D$ contains an increasing sequence of finite subgroups $D_{n}$, we also obtain a contradiction by examining $\chi_{D_{n}} * \chi_{D_{n}}$. We conclude that the center of $G$ must be compact.

Lemma 21. Suppose that $G$ is a connected, unimodular, metrizable, locally compact group, that $1 \leqslant p, q, r, s \leqslant \infty$, that $1 / p-1 / q>1 / r-1 / s \geqslant 0$, and that $L_{p}^{q}(G) \subset L_{r}^{s}(G)$. Suppose also that $G$ contains a normal abelian subgroup $N$. Then $N$ is compact.

Proof. Suppose $N$ is not compact. We shall reach a contradiction. Let $K$ be the maximal connected compact subgroup of $N$, which, being characteristic in $N$, is normal in $G$. By factoring out $K$ and applying Lemma 18, we can assume the connected component of $N$ is $R^{n}(n \geqslant 0)$. If $n=0, N$ is central (see [HR, 7.17]) and noncompact, which is impossible by Lemma 20. Therefore $n \geqslant 0$; being the connected component of $N, R^{n}$ is characteristic in $N$, so normal in $G$. We are reduced to considering the case where $G$ contains $R^{n}(n>0)$ as a normal subgroup.

Let $\pi$ be the canonical projection of $G$ onto $G / R^{n}$. G. Mackey [Mac, Lemma 1.1] tells us that there is a cross-section $\xi$ (i.e. a map $G / R^{n} \rightarrow G$ such that $\pi \xi$ is the identity) such that $\xi\left(G / R^{n}\right)$ is a Borel set and $\xi(K)$ is relatively compact if $K$ is compact. Let $K$ be a fixed compact subset of $G / R^{n}$.

We write $x^{g}$ for $g^{-1} x g$, and $B_{k}$ for the ball of radius $k$ in $R^{n}$. Evidently $\xi(K) B_{k}$ is a Borel set in $G$. Note

$$
\left[\xi(K) B_{k}\right]^{2}=\xi(K) B_{k} \xi(K) B_{k} \subset \xi(K)^{2} B_{k}^{\xi(K)} B_{k} .
$$

Automorphisms of $R^{n}$ are linear, and $\xi(K)$ is a relatively compact set, so $B_{k}^{\xi(K)} \leqslant$ $B_{c k}$ for some constant $c$. Then $\left[\xi(K) B_{k}\right]^{2} \subset \xi(K)^{2} B_{(c+1) k}$. Since $K^{2}$ is compact, $\xi\left(K^{2}\right)$ is relatively compact, as is $\xi(K)^{2}$. So $\xi\left(K^{2}\right)^{-1} \xi(K)^{2} \cap R_{n} \subset B_{b}$ for some finite $b$. It is now easily checked that $\xi(K)^{2} \subset \xi\left(K^{2}\right) B_{b}$, so that $\left[\xi(K) B_{k}\right]^{2}$ $C \xi\left(K^{2}\right) B_{b+(c+1) k}$.

Write $S_{k}$ for the Borel set $\xi(K) B_{k}$. Observe that $m\left(S_{k}\right)=m_{G / R^{n}}(K) m_{R^{n}}\left(B_{k}\right)$; so $m\left(S_{k}\right)$ can be made arbitrarily large. Note also

$$
m\left(S_{k}^{2}\right) \leqslant m_{G / R^{n}}\left(K^{2}\right) m_{R^{n}}\left(B_{b+(c+1) k}\right) \leqslant C m\left(S_{k}\right)
$$


for all sufficiently large $k$. We estimate the norms of the $\chi_{s_{k}}$ in $L_{r}^{s}(G)$ to obtain a contradiction via the closed graph theorem, as in so many preceding proofs.

Let $t$ be the number such that $1-1 / t=1 / p-1 / q$. Then (Lemma 19)

$$
\left\|\chi_{S_{k}}\right\|_{L_{p}^{q}} \leqslant\left\|\chi_{s_{k}}\right\|_{t}=m\left(S_{k}\right)^{1-1 / p+1 / q}
$$

However, $m\left(S_{k}\right) \chi_{s_{k}^{-1}} \leqslant \chi_{s_{k}} * \chi_{s_{k}^{-2}}$, so $m\left(S_{k}\right)\left\|\chi_{s_{k}^{-1}}\right\|_{s} \leqslant\left\|\chi_{s_{k}}\right\|_{L_{r}^{s}}\left\|\chi_{s_{k}^{-2}}\right\|_{r}$, i.e.

$$
m\left(S_{k}\right)^{1+1 / s} \leqslant\left\|\chi_{s_{k}}\right\|_{L_{r}^{s}} m\left(S_{k}^{2}\right)^{1 / r} .
$$

Applying the estimate obtained in the previous paragraph and remembering that $m\left(S_{k}\right)$ gets arbitrarily large as $k$ gets large, we conclude that $L_{p}^{q}(G) \not \subset L_{r}^{s}(G)$, a contradiction.

LEMMA 22. Suppose that $G$ is a unimodular Lie group, that $1 \leqslant p, q, r, s$ $\leqslant \infty$, that $1 / p>1 / r-1 / s \geqslant 0$, and that $L_{p}^{q}(G) \subset L_{r}^{s}(G)$. Then the radical of $G$ is compact.

Proof. The radical $R$ of $G$ is the maximal connected solvable normal subgroup of $G$. There is a finite series

$$
R=R^{(0)} \triangleright R^{(1)} \triangleright R^{(2)} \triangleright \cdots \triangleright R^{(n-1)} \triangleright R^{(n)}=\{e\},
$$

where each $R^{(j+1)}$ is the topological commutator subgroup of $R^{(j)}$, so characteristic in $R$, and normal in $G$; also, $R^{(j)} / R^{(j+1)}$ is abelian.

If $R$ is noncompact, at least one factor $R^{(j)} / R^{(j+1)}$ is noncompact. Suppose that $R^{(j+1)}$ is compact but $R^{(j)}$ is not. By Lemma $18, L_{p}^{q}\left(G / R^{(j+1)}\right) \subset$ $L_{r}^{s}\left(G / R^{(j+1)}\right)$, but $R^{(j)} / R^{(j+1)}$ is a noncompact, normal, abelian subgroup of $G / R^{(j+1)}$, which is impossible by Lemma 21 . We conclude that $R$ must be compact.

Proof of Theorem 15 . We suppose that $G$ is a unimodular locally compact group, and that $L_{p}^{q}(G) \subset L_{r}^{s}(G)$ where $1 \leqslant p, q, r, s \leqslant \infty$ and $1 / p-1 / q>$ $1 / r-1 / s \geqslant 0$. By Yamabe's theorem, $G$ has an open subgroup $G_{1}$ with a compact normal subgroup $N$ such that $G_{1} / N$ is a connected Lie group. By Lemma 18, $L_{p}^{q}\left(G_{1} / N\right) \subset L_{r}^{s}\left(G_{1} / N\right)$; so the radical $R / N$ of $G_{1} / N$ is compact by Lemma 22. Then $R$ is a compact normal subgroup of $G_{1}$, and $G_{1} / R$ is a semisimple Lie group. Applying Lemmas 18 and 20 , we conclude that $G_{1} / R$ has finite centre, as required.

Proof of TheOREM 16 . We suppose that $G$ is a unimodular, locally compact group, and that $L_{p}^{q}(G) \subset L_{r}^{s}(G)$, where $1 \leqslant p, q, r, s \leqslant \infty$ and $1 / p-1 / q>$ $1 / r-1 / s \geqslant 0$. Suppose that $G_{1}$ is a compact open subgroup of $G$. Since $m\left(G_{1}\right) \chi_{G_{1}}=\chi_{G_{1}} * \chi_{G_{1}},\left\|\chi_{G_{1}}\right\|_{L_{r}^{s}} \geqslant m\left(G_{1}\right)^{1+1 / 5-1 / r}$. However, if $t$ is the number such that $1-1 / t=1 / p-1 / q$, then

$$
\left\|\chi_{G_{1}}\right\|_{L_{q}^{p}} \leqslant\left\|\chi_{G_{1}}\right\|_{t}=m\left(G_{1}\right)^{1+1 / q-1 / p}
$$


An application of the closed graph theorem now shows that there is a constant $C$ such that $m\left(G_{1}\right) \leqslant C$ for every compact open subgroup $G_{1}$ of $G$. It follows that every compact open subgroup of $G$ is contained in a maximal compact open subgroup of $G$, because the Haar measure of all compact open subgroups is uniformly bounded.

Suppose now that $K$ is a compact open subgroup of $G$, that $x \in G \backslash K$, and that $\left\{x^{n}\right\} \cap K=\{e\}$ for all $n$. If any relations $x^{n} K=K x^{m}$ hold $(m, n \neq 0)$, we reach a contradiction as follows. Consider the function $\Sigma_{k=1}^{N} L\left(x^{k n}\right) \chi_{K}$. On the one hand, by Lemma 19 ,

$$
\left\|\sum_{1}^{N} L\left(x^{k n}\right) \chi_{K}\right\|_{L_{p}^{q}} \leqslant[N \cdot m(K)]^{1+1 / q-1 / p} .
$$

On the other hand,

$$
\left[\sum_{k=1}^{N} L\left(x^{k n}\right) \chi_{K}\right] *\left[\sum_{j=1}^{N} R\left(x^{j m}\right) \chi_{K}\right]=\sum_{1}^{N} \sum_{1}^{N} L\left(x^{k n}\right) L\left(x^{j n}\right) \chi_{K},
$$

so that, for some constant $C$,

$$
\left\|\sum_{1}^{N} L\left(x^{k n}\right) \chi_{K}\right\|_{L_{r}^{s}} \geqslant C[N \cdot m(K)]^{1+1 / s-1 / r} .
$$

Application of the closed graph theorem yields the required contradiction.

REMARK 8. The following result appears likely: if $G$ is an amenable, unimodular, noncompact, locally compact group (i.e. if there exists an invariant mean on $L^{\infty}(G)$; F. Greenleaf [Gre] gives a good discussion of amenability), and $1 / p-1 / q>1 / r-1 / s \geqslant 0$, then $L_{p}^{q}(G)$ is not included in $L_{r}^{s}(G)$. It is certainly true if $r=s$, or if $G$ is near-connected. For in the first case, $L^{t}(G) \subset$ $L_{r}^{s}(G)$ for some $t$ greater than one, but any positive locally integrable function in $L_{r}^{s}(G)$ belongs to $L^{1}(G)$, so that $G$ must be compact; in the second case, we combine Theorem 15 with the known fact that all amenable, connected, semisimple, Lie groups are compact.

REMARK 9. If $G$ is a connected, semisimple, Lie group with finite centre, then probably $L^{t}(G) \subset L_{r}^{s}(G)$ if $1 / s<1 / t \leqslant 1-1 / r+1 / s$ and $1 / r+1 / s \geqslant 1$. For certain such groups $(\operatorname{SL}(2, R), \operatorname{SL}(2, C)$, for example) it is known that $L^{p}(G) * L^{2}(G) \subset L^{2}(G)$ if $1 \leqslant p<2-$ see [KS], [Lip 1], [Lip 2], [Lip 3], and recent results [EL] support this hypothesis in general. Rewriting this inclusion as $L^{2}(G) * L^{p}(G) \subset L^{2}(G)$ and using multilinear complex interpolation between this result and the known inclusions $L^{1}(G) * L^{1}(G) \subset L^{1}(G), L^{1}(G) * L^{\infty}(G)$ $\subset L^{\infty}(G)$ proves the claim when $L^{2}(G) * L^{p}(G) \subset L^{2}(G)$.

REMARK 10. Despite the above remark, $L^{2}(G) \subset L_{2}^{2}(G)$ if and only if $G$ is compact [Ric]. Another result is the same vein is due to $N$. Lohoue [personal 
communication]: if $G$ is a near-connected locally compact group, $1<p<2$, and $L^{p}(G) \subset L_{p}^{p}(G)$, then $G$ is compact.

REMARK 11. If $G$ is a noncompact semisimple Lie group with finite centre, and if all measures of the form $\Sigma_{1}^{\infty} a_{i} \epsilon\left(x_{i}\right)$, where $\Sigma_{1}^{\infty}\left|a_{i}\right|^{p}<\infty$ and $\left\{x_{i}\right\}$ is discrete, belong to $L_{2}^{2}(G)$, then $p=1$. This is proved using results of Herz [Her 2], which show that $L_{2}^{2}(Z)$ can be identified with the subspace of $L_{2}^{2}(G)$ of operators "supported" by $Z$, where $Z$ is a discrete subgroup of $G$ isomorphic to the integers.

Appendix 2. Convolution operators between Lorentz spaces. Recall that, if $s \leqslant t$, then $L^{p, s}(G) \subset L^{p, t}(G)$. We therefore have the inclusion

$$
L_{p, q}^{p, s} \subset L_{p, r}^{p, t} \text { whenever } q \geqslant r \text { and } s \leqslant t .
$$

We now show that many of these inclusions are strict if the group $G$ is infinite and $p \neq 1,2, \infty$. In particular, we show that, in this situation, $L_{p, 1}^{p, \infty} \neq L_{p, p}^{p, \infty}$, that is, that there exist convolution operators of restricted weak type $(p, p)$ that are not of weak type $(p, p)$. Unfortunately, our methods do not tell us whether, when $p>2$, there must exist convolution operators of weak type $(p, p)$ that are not of strong type $(p, p)$.

Just as in $\S 3$, we prove our results by estimating the norms, in the various spaces $L_{p, q}^{p, s}$, of sums of translates of Rudin-Shapiro measures. It seems best, however, to change our convention concerning Rudin-Shapiro measures, by replacing left translation throughout by right translation, much as in $\S 5$. That is, we still let $\rho_{0}=\sigma_{0}=\epsilon(e)$, but now, given $\rho_{n}$ and $\sigma_{n}$ both supported by a finite set $S_{n}$, we choose $x_{n}$ in $G$ so that the set $S_{n} x_{n}$ its disjoint from $S_{n}$, and we let

$$
\rho_{n+1}=\rho_{n}+R\left(x_{n}\right) \sigma_{n},
$$

and

$$
\sigma_{n+1}=\rho_{n}-R\left(x_{n}\right) \sigma_{n}
$$

These measures differ from those defined in $\S 2$, unless the $x_{n}$ commute. Much as in $\$ 2$, however, the new measures can be represented in the form

$$
\rho_{n}=\sum_{k=1}^{2^{n}} r_{k} \epsilon\left(y_{R}\right) \text { and } \sigma_{n}=\sum_{k=1}^{2^{n}} s_{k}^{n} \epsilon\left(y_{n}\right)
$$

although the sequence $\left(y_{k}\right)_{k=1}^{\infty}$ may differ from the one that arose when we used left translation.

We continue to identify measures $\mu$ with convolution operators $g \longmapsto$ $\mu * g$. It is no longer obvious that Lemma 1 holds; indeed, it seems doubtful that identity (10) of $\S 2$ holds if the $x_{k}$ do not commute. Nevertheless, inequality 
(11) and Lemma 1 still hold. The quickest way to see this is to observe that the reflected measures $\rho_{n}^{\vee}$ and $\sigma_{n}^{\vee}$ are Rudin-Shapiro measures constructed using the left translation operators $L\left(x_{0}^{-1}\right), L\left(x_{1}^{-1}\right), \ldots$. Now

$$
\left\|\rho_{n}\right\|_{L_{p}^{p}}=\left\|\rho_{n}\right\|_{L} p_{p^{\prime}}^{\prime} \text { and }\left\|\sigma_{n}\right\|_{L p}=\left\|\sigma_{n}\right\|_{L} \|_{p^{\prime}}^{\prime}
$$

therefore, the estimates (8) and (9) continue to hold for the new measures $\rho_{n}$ and $\sigma_{n}$.

For later use, we need an identity that implies, incidentally, that $\rho_{n}$ can itself be regarded as a Rudin-Shapiro measure constructed using the left translation operators $L\left(x_{i}\right)$ in the order $L\left(x_{n-1}\right), L\left(x_{n-2}\right), \ldots, L\left(x_{1}\right), L\left(x_{0}\right)$. To this end, we fix an integer $N>0$, and define measures $\left\{\eta_{n}\right\}_{n=0}^{N}$ and $\left\{\zeta_{n}\right\}_{n=0}^{N}$, by letting $\eta_{0}=\zeta_{0}=\epsilon(e)$, and, given $\eta_{n}$ and $\zeta_{n}$, letting

and

$$
\eta_{n+1}=\eta_{n}+L\left(x_{N-n-1}\right) \zeta_{n} \text {, }
$$

The point of these definitions is that, if $m+n=N-1$, then

$$
\rho_{N}=\rho_{m} * \eta_{n}+\left(R\left(x_{m}\right) \sigma_{m}\right) * \zeta_{n} \text {. }
$$

This identity is obvious if $n=0$, and follows for other values of $n$ by an inductive argument that depends on the fact that $[R(x) \mu] * \nu=\mu *[L(x) \nu]$; the corresponding identity for Rudin-Shapiro polynomials appears, in a disguised form, in [Bri, Theorem 1]. Setting $m=0$ in formula (36) we see that $\rho_{N}=$ $\eta_{N-1}+L\left(x_{0}\right) \zeta_{N-1}=\eta_{N}$.

Continue to fix $N$, let $S_{N}$ denote the support of $\rho_{N}$, and let $T=$ $\left[S_{N} \cup S_{N}^{-1}\right]^{3}$. Proceeding inductively, choose elements $\left\{v_{n}\right\}_{n=0}^{N-1}$ and $\left\{w_{n}\right\}_{n=0}^{N-1}$ of $G$ so that each $v_{n}$ lies outside the sets $\bigcup_{m<n} T v_{m}$ and $\bigcup_{m<n} T w_{m}$, while each $w_{n}$ lies outside these sets and outside $T v_{n}$ as well; this is possible if $G$ is infinite. Fix $p$ in the interval $(1,2)$, and let

$$
v_{N}=\sum_{m=0}^{N-1} 2^{-m / p}\left\{R\left(\nu_{m}\right) \rho_{m}+R\left(w_{m}\right) R\left(x_{m}\right) \sigma_{m}\right\} .
$$

Finally, let $\nu_{N}^{\vee}$ be the reflection of $\nu_{N}$.

LEMMA 23. Let $1 \leqslant r \leqslant s \leqslant \infty$, let $u=\min \left(s, r^{\prime}\right)$, and let $1 / q=1 / s+$ $1 / r$. Then the norm of $\nu_{N}$ in $L_{p, r}^{p, s}$ is at least $C N^{1 / u}$, and at most $D N^{1 / q}$, for positive constants $C$ and $D$ that depend only on $N$. The same estimates hold for the norm of $\nu_{N}$ in $L_{p^{\prime}, s^{\prime}}^{p^{\prime}, r^{\prime}}$.

Proof. Write $1 / p=(1-\theta) / 2+\theta / 2$. Now $v_{N}$ belongs to $L_{2}^{2}(G) \cap L_{1}^{1}(G)$ and hence to the intermediate space $\left(L_{2}^{2}(G), L_{1}^{1}(G)\right)_{\theta, q}$. Exactly as in $\S 3$, we can 
estimate $K\left(t ; v_{N}\right)$, and show that $\left\|\nu_{N}\right\|_{\theta, q}=O\left(N^{1 / q}\right)$ as $N \rightarrow \infty$. By Theorem 3 , the norm of $\nu_{N}$ in $L_{p, r}^{p, s}$ is $O\left(N^{1 / q}\right)$ as $N \rightarrow \infty$. This is the desired upper bound.

To get the lower bound, we suppose first that $1 / r+1 / s \geqslant 1$; then $u=s$. The assumptions concerning the sequences $\left(v_{n}\right)_{n=0}^{N-1}$ and $\left(w_{n}\right)_{n=0}^{N-1}$ guarantee that the various terms in the series (37) have disjoint support. Thus $v_{N}$ is a sum of $\sum_{m=0}^{N-1} 2 \cdot 2^{m}=2\left(2^{N}-1\right)$ disjoint point-masses. Choose a compact neighbourhood $K$ of the identity so that, if $x$ and $y$ are distinct points in the support of $v_{N}$, then the sets $x K$ and $y K$ are disjoint. Let $f$ be the characteristic function of $K$; then $\|f\|_{p, r}$ is essentially $m(K)^{1 / p} \quad$ [SW 2, p. 192]. Let $g=\nu_{N} * f$, and let $g^{*}$ be the nonincreasing rearrangement of $g$ on $(0, \infty)$; then

$$
g^{*}(u)= \begin{cases}2 & \text { if } 0<u<2 m(K), \\ 2^{-m / p} & \text { if } 2\left(2^{m}-1\right) m(K) \leqslant u<2\left(2^{m+1}-1\right) m(K), \\ 0 & \text { if } u \geqslant 2\left(2^{N}-1\right) m(K) .\end{cases}
$$

Now $\|g\|_{p, s}$ is essentially $\left\{\int_{0}^{\infty}\left[u^{1 / p} g^{*}(u)\right]^{s} d u / u\right\}^{1 / s}$ if $s<\infty$, and $\sup _{u} u^{1 / p} g^{*}(u)$ if $s=\infty$. It follows that $\|g\|_{p, s}$ is essentially $N^{1 / s} m(K)^{1 / p}$. Therefore, the norm of $\nu_{N}$ in $L_{p, r}^{p, s}$, which is at least $\|g\|_{p, s} /\|f\|_{p, r}$, must be bounded below by $C N^{1 / s}$, for a positive constant $C$ that is independent of $N$.

Now suppose that $1 / r+1 / s<1$; then $u=r^{\prime}$. Define a discrete measure $\beta_{N}$ by letting

$$
\left.\beta_{N}=\sum_{n=0}^{N-1} 2^{-n / p}\left\{L\left(v_{N-n-1}^{-1}\right) m_{n}+L\left(w_{N-n-1}^{-1}\right)\right\}_{n}\right\} .
$$

Form the product $\nu_{N} * \beta_{N}$. If $m+n=N-1$, then by identity (36), the terms

$$
\left[R\left(v_{m}\right) \rho_{m}\right] *\left[L\left(v_{m}^{-1}\right) m_{n}\right] \text { and }\left[R\left(w_{m}\right) R\left(x_{m}\right) \sigma_{m}\right] *\left[L\left(w_{m}^{-1}\right) \zeta_{n}\right]
$$

are supported by $S_{N}$. The choice of $\left\{v_{n}\right\}$ and $\left\{w_{n}\right\}$ guarantees that all other terms appearing in the expansion of the product $\nu_{N} * \beta_{N}$ have supports disjoint from $S_{N}$; let $\alpha_{N}$ denote the sum of these other terms. Then, by formula (36), $\nu_{N} * \beta_{N}=N 2^{-(N-1) / p} \rho_{N}+\alpha_{N}$. Choose a compact neighbourhood $K$ of the identity, with characteristic function $f$, so that if $x$ and $y$ are distinct points in the support of $\rho_{N}$ or $\beta_{N}$, then the sets $x K$ and $y K$ are disjoint, and so that the functions $\rho_{N} * f$ and $\alpha_{N} * f$ have disjoint support. Let $g=\beta_{n} * f$, and let

$$
h=v_{N} * g=\left[N 2^{-(N-1) / p_{\rho_{n}}}+\alpha_{N}\right] * f
$$

Then, as above, $\|g\|_{p, r}$ is essentially $N^{1 / r} m(K)^{1 / p}$. Because the functions $\rho_{N} * f$ and $\alpha_{N} * f$ have disjoint support, 


$$
\begin{aligned}
\|h\|_{p, s} & \geqslant N 2^{-(N-1) / p}\left\|\rho_{N} * f\right\|_{p, s} \geqslant N 2^{-(N-1) / p}\left\|\rho_{N} * f\right\|_{p, \infty} \\
& \geqslant N 2^{-(N-1) / p}\left[2^{N} m(K)\right]^{1 / p}=2^{1 / p} N m(K)^{1 / p} .
\end{aligned}
$$

This shows that the norm of $\nu_{N}$ in $L_{p, r}^{p, s}$, which is at least $\|h\|_{p, s} /\|g\|_{p, r}$, is bounded below by $C N^{1 / r^{\prime}}$, for a positive constant $C$ that is independent of $N$.

That similar estimates hold for the norm of $\nu_{N}^{v}$ in $L_{p^{\prime}, s^{\prime}}^{p^{\prime}, r^{\prime}}$ follows by a simple duality argument based on two facts. First,

$$
\int_{G}\left(\nu_{N} * f\right)(x) g(x) d x=\int_{G} f(x)\left[\nu_{N}^{\vee} * f(x)\right] d x,
$$

for all $f$ and $g$ in $C_{c}(G)$. Second, if $h \in L^{p, t}(G)$, then $\|h\|_{p, t}$ is essentially equal to

$$
\sup \left\{\left|\int_{G} f(x) h(x) d x\right|: f \in C_{c}(G),\|f\|_{p^{\prime}, t^{\prime}}=1\right\} .
$$

This completes the proof of the lemma.

We now use the lemma to show that many of the inclusions (31) are strict.

THEOREM 24. Let $G$ be an infinite, locally compact group. Let $1<p<$ 2 or $2<p<\infty$. If $1<q<2$, and $q \leqslant r<t \leqslant q^{\prime}$, then the inclusion

$$
L_{p, q}^{p, r} \subset L_{p, q}^{p, t}
$$

is strict. If $2<r \leqslant \infty$, and $r^{\prime} \leqslant q<s \leqslant r$, then the inclusion

$$
L_{p, s}^{p, r} \subset L_{p, q}^{p, r}
$$

is strict.

Proof. If inclusion (38) is not strict, then the norms in the two spaces must be equivalent. We now show that these norms are, in fact, not equivalent. Suppose first that $1<p<2$, and that $q=1$. We know by Lemma 23 that the norm of $\nu_{N}$ in $L_{p, 1}^{p, r}$ is at least $C N^{1 / r}$, while its norm in $L_{p, 1}^{p, t}$ is at most $D N^{1 / t}$. Hence, if $1 \leqslant r<t \leqslant \infty$, then the norms in the spaces $L_{p, 1}^{p, r}$ and $L_{p, 1}^{p, t}$ are not equivalent.

If $1<q<2$, then $L^{p, q}(G)$ is reflexive [Hun, p. 262]. Let $0 \leqslant \theta \leqslant 1$, and $u=q /(1-\theta)$. Then in the complex interpolation method [Cal, pp. 124128], we have that $\left[L^{p, q}(G), L^{p, \infty}(G)\right]_{\theta}=L^{p, u}(G)$, with an equivalent norm. Renorm the spaces $L^{p, u}(G)$, using the intermediate norm arising from the complex method, and give the spaces $L_{p, q}^{p, u}$ the new operator norm. For each positive integer $N$, and each point $\phi$ in the interval $[0,1 / q)$, let $f_{N}(\phi)$ be the logarithm of the norm of $\nu_{N}$ in the space $L_{p, q}^{p, 1 / \phi}$. Then $f_{N}$ is a convex function. Suppose that $q \leqslant t<q^{\prime}$; then by Lemma 23 , we can make the difference $f_{N}(1 / t)-f_{N}(0)$ 
as large as we like, simply by taking $N$ large. Now, if also $q \leqslant r<t$, then, by the convexity of $f_{N}$, we can make the difference $f_{N}(1 / r)-f_{N}(1 / t)$ as large as we like. This shows, for such indices $q, r$, and $t$, that the norms in the spaces $L_{p, q}^{p, r}$ and $L_{p, q}^{p, t}$ are not equivalent; hence the norms in $L_{p, q}^{p, r}$ and $L_{p, q}^{p, q^{\prime}}$ are also not equivalent. Therefore, inclusion (38) is strict whenever $q \leqslant r<t \leqslant q^{\prime}$, and $1<$ $p<2$.

If $p>2$, then we repeat the argument above, with $\nu_{N}^{v}$ in place of $\nu_{N}$, and we conclude again that inclusion (38) is strict whenever $q \leqslant r<t \leqslant q^{\prime}$. Finally, we can prove the strictness of inclusion (39) by a similar application of Lemma 23 , or by a duality argument starting with the strictness of inclusion (38). This completes the proof of the theorem.

COROLlary 25. If $G$ is infinite, and $p \neq 1,2, \infty$, then there exists a convolution operator on $G$ that belongs to $L_{p, 1}^{p, \infty}$ but to no other space $L_{p, q}^{p, r}$.

Proof. It suffices to find an element of $L_{p, 1}^{p, \infty}$ that does not belong to

$$
\left(\bigcup_{r<\infty} L_{p, 1}^{p, r}\right) \cup\left(\bigcup_{q>1} L_{p, q}^{p, \infty}\right),
$$

because $L_{p, q}^{p, r} \subset L_{p, 1}^{p, r} \cap L_{p, q}^{p, \infty}$ for all $q$ and $r$. Now, if $r<\infty$, then the inclusion $L_{p, 1}^{p, r} \subset L_{p, 1}^{p, \infty}$ is strict. The method of the gliding hump [EP] yields an element $S$ of $L_{p, 1}^{p, \infty}$ that does not belong to $\bigcup_{r<\infty} L_{p, 1}^{p, r}$. Similarly there exists an operator $T$ that belongs to $L_{p, 1}^{p, \infty}$ but not to $\bigcup_{q>1} L_{p, q}^{p, \infty}$. Then one of the operators $S, T$, or $S+T$ has the desired properties.

COROLLARY 26. If $G$ is infinite and compact, and if $p \neq 1,2, \infty$, then the inclusion $L_{p, q}^{p, r} \subset L_{p, q}^{p, t}$ is strict whenever $q<2$ and $1 \leqslant r<t \leqslant q^{\prime}$, and the inclusion $L_{p, s}^{p, r} \subset L_{p, q}^{p, r}$ is strict whenever $r>2$ and $r^{\prime} \leqslant q<s \leqslant \infty$.

Proof. Consider the function $f_{N}$ defined in the proof of the theorem. If $f_{N}$ were finite on the interval $(1 / q, 1)$, then the argument given above would show that inclusion (38) is strict whenever $q<2$ and $1 \leqslant r<t \leqslant q^{\prime}$. In fact, no sum of finitely many point masses can belong to $L_{p, q}^{p, r}$ if $r<q$, and $f_{N}$ must therefore take the value $+\infty$ on the interval $(1 / q, 1)$. Using approximate identities, however, we can replace each measure $\mu_{N}$ by a trigonometric polynomial $g_{N}$ so that when $f_{N}$ is redefined appropriately, we can still make the difference $f_{N}(1 / q)$ $-f_{N}(0)$ as large as we like simply by taking $N$ large. Now the map $h \mapsto g_{N} * h$ takes $L^{p, q}(G)$ into $L^{\infty}(G)$; since $G$ is compact, this map belongs to $L_{p, q}^{p, 1}$. Hence the new functions $f_{N}$ are finite in the whole interval $[0,1]$, and we conclude, as above, that inclusion (38) is strict whenever $q<2$ and $1 \leqslant r<t \leqslant q^{\prime}$.

Similarly, the inclusion $L_{p, s}^{p, r} \subset L_{p, q}^{p, r}$ is strict whenever $r>2$ and $r^{\prime} \leqslant q<s \leqslant \infty$.

REMARK 12. If $G$ is not compact, then the spaces $L_{p, q}^{p, r}$, with $r<q$, are 
all trivial. It has already been shown [Blo] that $L_{p, q}^{p, r}$ contains no positive operators other than the 0-operator; if $L_{p, q}^{p, r}$ contains any nonzero operator, then, by convolution with a suitable approximate identity, $L_{p, q}^{p, r}$ contains an operator $h \rightarrow$ $f * h$ where $f$ is a nontrivial continuous function. We can assume that $f$ is real. Choose $g$ in the Fourier algebra $A(G)$ so that $f \cdot g$ is positive somewhere and negative nowhere. Then convolution with $f \cdot g$ is a nontrivial, positive operator in $L_{p, q}^{p, r}$, a contradiction.

REMARK 13. There are various ways in which Theorem 24 is not as sharp as we would like. In particular, we do not know whether the inclusion $L_{p, p}^{p, p} \subset$ $L_{p, p}^{p, \infty}$ is strict when $G$ is infinite and $p>2$. Under the analogy between the spaces $L^{p, r}(G)$ and the spaces $\ell^{r}$, the inclusion above corresponds to the inclusion $\ell_{p}^{p} \subset \ell_{p}^{\infty}$. Now, on a discrete group, $\ell_{p}^{\infty}=\ell^{p^{\prime}}$; it is not known whether there exists an infinite, discrete group such that $\ell^{p^{\prime}} \subset \ell_{p}^{p}$, for an index $p$ in the interval $(2, \infty)$. Any such group could have no infinite abelian subgroup, and, by Theorem 16, there would be a bound on the order of its finite subgroups.

REMARK 14. Comparing Lemma 23 with Lemma 6, we conjecture that some measure like $\nu_{N}$ should have norm at most $D N^{1 / t}$, rather than $D N^{1 / q}$, in the space $L_{p, r}^{p, s}$; the way to prove this would be to deal first with the special case of $L_{p, 2}^{p, 2}$, and then use convexity arguments much as in the proof of Lemma 6 . The existence of such a sequence of measures would imply, for instance that $L_{p}^{p}(G) \not \subset L_{p, 1}^{p, q}$ when $1<p<2$ and $1 \leqslant q<p$. Misha Zafran has a proof of this noninclusion for the integer group [private communication].

REMARK 15. As in $\S 6$, the analogues of Lemma 23 and Theorem 24 also hold for the spaces $L_{p, q}^{p, s}$ of operators that commute with left translation rather than right translation. It would be desirable to also have the analogues of these results for the corresponding spaces of central convolution operators, that is those operators that commute with both left and right translation. If $Z(G)$, the centre of $G$, is infinite, then these results do hold, because we can choose the various Rudin-Shapiro measures to be supported by $Z(G)$; if $Z(G)$ is finite, however, then our methods do not yield any information about inclusions between spaces of central convolution operators.

Appendix 3. Coincidence of some spaces of convolution operators. This part of the paper is independent of the rest, although we use the same notation. We deal with operators that commute with right translation, but the results also hold, with similar proofs, for operators that commute with left translation. Our aim is to generalize the following known result to noncompact groups.

TheOREM 27. Let $G$ be compact, let $1 \leqslant p \leqslant 2$, and let $q<p$. Then $L_{p}^{q}(G)=L_{p, p}^{p, \infty}$, with equivalence of norms. 
This was first proved for the circle group by Stein [Ste 2, §16]. Subsequently, Sawyer [Saw] used the same method to prove that, if $G$ is compact and $1 \leqslant p \leqslant 2$, then every convolution operator, with domain $L^{p}(G)$, that is continuous in measure must be of weak type $(p, p)$; Theorem 27 follows easily from this result. A simplified account of Sawyer's method appears in [Gar, §1.2]. Later, Doss [Dos] rediscovered Theorem 27, for compact abelian groups, with $1 \leqslant q<p \leqslant 2$, deriving the result directly from Stein's original theorem on limits of sequences of operators [Ste 2].

If $q<p$, and $G$ is not compact, then $L_{p}^{q}(G)=\{0\}$, and the inclusion $L_{p}^{q}(G) \subset L_{p, p}^{p, \infty}$ holds trivially. The correct generalization of Theorem 27 , however, involves spaces of locally integrable functions. For $p<\infty$ denote the space $L^{p}(G)+L^{\infty}(G)$ by $L_{\text {lar }}^{p}$. Define a norm on $L_{\text {lar }}^{p}$ as follows. Fix a positive number $a$, with the added requirement when $G$ is discrete that $a \geqslant m(\{e\})$. Then let

$$
\|f\|_{p, \mathrm{lar}}=\sup _{K}\left(\int_{K}|f|^{p}\right)^{1 / p}, \quad m(K) \leqslant a ;
$$

that is, if $f^{*}$ is the nonincreasing rearrangement of $f$, then

$$
\|f\|_{p, \text { lar }}=\left[\int_{0}^{a} f^{*}(t)^{p} d t\right]^{1 / p}
$$

Relative to this norm, $L_{\text {lar }}^{p}$ is a Banach space. Similarly, let $L_{\text {lar }}^{p, \infty}$ consist of all functions $f$ such that $\sup _{t<a} t^{1 / p} f^{*}(t)<\infty$.

THEOREM 28. Let $G$ be a locally compact group that is not compact. Let $1 \leqslant p<\infty$, and let $q<p$. Then the space of bounded convolution operators from $L^{p}(G)$ to $L_{\mathrm{lar}}^{q}$ coincides with the space of bounded convolution operators from $L^{p}(G)$ to $L_{\text {lar }}^{p, \infty}$.

Proof. If an operator is bounded from $L^{p}(G)$ to $L_{\text {lar }}^{p, \infty}$, then it is bounded from $L^{p}(G)$ to $L_{\text {lar }}^{q}$, because $L_{\text {lar }}^{p, \infty}$ is continuously imbedded in $L_{\text {lar }}^{q}(G)$.

To prove the converse inclusion, we modify an argument due to Coifman [Coi]. Suppose that $T$ is a bounded convolution operator from $L^{p}(G)$ to $L_{\text {lar }}^{q}$, with norm $D$. Let $f \in C_{c}(G)$. Fix a set $E$ with $0<m(E) \leqslant a$, and suppose that $|T f(x)|>\lambda>0$ for all $x$ in $E$. Let $N$ be the smallest integer for which $N m(E)$ $>a$. Choose elements $\left\{x_{n}\right\}_{n=1}^{N}$ of the kernel of the modular function so that the translates $R\left(x_{n}\right) f$ have disjoint support, and so that $m\left(\bigcup_{n=1}^{N} E x_{n}\right)>a$; this is possible because the kernel of the modular function is not compact. Let $K$ be a compact subset of $\bigcup_{n=1}^{N} E x_{n}$ such that $a / 2<m(K) \leqslant a$.

Define the Rademacher functions $\left\{r_{n}\right\}_{n=0}^{\infty}$ as in [Ste 3, p. 104]. For each point $t$ in the unit interval $[0,1]$, let $F_{t}$ be the function on $G$ given by $F_{t}=$ $\sum_{n=1}^{N} r_{n}(t) R\left(x_{n}\right) f$, and let $g_{t}=T\left(F_{t}\right)$. Then 


$$
\left.\int_{K}\left|g_{t}(x)\right|^{q} d x \leqslant\left\{D \int_{G}\left|F_{t}(x)\right|^{p} d x\right]^{1 / p}\right\}^{q} .
$$

Because the functions $R\left(x_{n}\right) f$ have disjoint support, and because $\left\|R\left(x_{n}\right) f\right\|_{p}=$ $\|f\|_{p}$ for all $n$, the right side of inequality (40) is equal to $\left(D N^{1 / p}\|f\|_{p}\right)^{q}$, for all $t$. Now, by Fubini's theorem,

$$
\int_{0}^{1} \int_{K}\left|g_{t}(x)\right|^{q} d x d t=\int_{K} \int_{0}^{1}\left|g_{t}(x)\right|^{q} d t d x
$$

and, by a standard property of Rademacher series [Ste 3, p. 278], the inner integral on the right is at least $C_{q}^{-q}\left(\Sigma_{n=1}^{N}\left|T f\left(x x_{n}^{-1}\right)\right|^{2}\right)^{q / 2}$, for a constant $C_{q}$ that is independent of $x$. Since each point $x$ of $K$ belongs to $E x_{n}$ for some $n$, and $\left|T f\left(x x_{n}^{-1}\right)\right|>\lambda$ for this value of $n$, the left side of inequality (40) is at least $\left(\lambda / C_{q}\right)^{q} m(K)$. Hence

$$
(a / 2)^{1 / q}\left(\lambda / C_{q}\right) \leqslant D N^{1 / p}\|f\|_{p} .
$$

But $m(E)<2 a / N$, so that

$$
\lambda m(E)^{1 / p} \leqslant 2^{1 / p+1 / q} a^{1 / p-1 / q} C_{q} D\|f\|_{p} .
$$

Therefore,

$$
\sup _{t<a} t^{1 / p}(T f)^{*}(t) \leqslant 2^{1 / p+1 / a} a^{1 / p-1 / q} C_{q} D\|f\|_{p} .
$$

This proves that $T$ is a bounded operator from $L^{p}(G)$ to $L_{\text {lar }}^{p, \infty}$, and completes the proof of the theorem.

Our estimate (43) for the weak-type $(p, p)$ norm of $T$ depends on the parameter $a$, and on $D$, which also depends on $a$. To characterize the space of convolution operators of weak type $(p, p)$, we consider the dependence of $D$ on $a$.

COROLlARY 29. Let $G$ be a locally compact group that is not compact. Let $1 \leqslant p<\infty$, and let $q<p$. Suppose that $T$ is a bounded convolution operator from $L^{p}(G)$ to $L_{\text {lar }}^{q}$, with norm $D_{a}$. Then $T$ is of weak type $(p, p)$ if and only if

$$
D_{a}=O\left(a^{1 / q-1 / p}\right) \text { as } a \rightarrow \infty .
$$

Proof. If $T \in L_{p, p}^{p, \infty}$, then

Hence

$$
\sup _{t} t^{1 / p}(T f)^{*}(t) \leqslant\|T\|\|f\|_{p}, \text { for all } f \text { in } L^{p}(G) .
$$

$$
\begin{aligned}
{\left[\int_{0}^{a}(T f)^{*}(t)^{q} d t\right]^{1 / q} } & \leqslant\|T\|\|f\|_{p}\left(\int_{0}^{a} t^{-q / p} d t\right)^{1 / q} \\
& =\|T\|\|f\|_{p} O\left(a^{1 / q-1 / p}\right),
\end{aligned}
$$

and statement (44) follows. 
Suppose, on the other hand, that

$$
D_{a} \leqslant C a^{1 / q-1 / p},
$$

for some constant $C$, and all large values of $a$. Let $f \in C_{c}(G)$, let $\lambda>0$, and let $E$ be a set of positive, but finite, measure on which $|T f|>\lambda$. Choose the parameter $a$ so large that inequality (45) holds, and so that $a \geqslant m(E)$. Then, by inequality (42),

$$
\lambda m(E)^{1 / p} \leqslant 2^{1 / p+1 / q} C \cdot C_{q}\|f\|_{p} .
$$

This proves that $T$ is of weak type $(p, p)$, and completes the proof of the corollary.

Theorem 27 provides an infinite family of distinct Banach spaces, on any infinite compact group, namely the $L^{q}$ spaces with $q<p$, such that the space of bounded convolution operators from $L^{p}$ to any of these spaces coincides with $L_{p, p}^{p, \infty}$. A similar family of spaces exists on any noncompact, nondiscrete group. If $q<p$, let $C^{p, q}(G)$ consist of all functions $f$ on $G$ such that

$$
\left[\int_{0}^{a}\left|f^{*}(t)\right|^{q}\right]^{1 / q}=O\left(a^{1 / q-1 / p}\right) \quad \text { as } a \rightarrow \infty .
$$

To make $C^{p, q}(G)$ a Banach space, we identify functions that differ only on locally null sets, and we define the norm of each element $f$ in $C^{p, q}(G)$ to be

$$
\sup _{a \geq 1} a^{1 / p-1 / q}\left[\int_{0}^{a} f^{*}(t)^{q} d t\right]^{1 / q}
$$

It is easy to verify that $L^{p, \infty}(G) \subset C^{p, q}(G)$ for all $q<p$, and that $C^{p, q}(G) \subset$ $C^{p, r}(G)$ if $r<q$; moreover, these inclusions are strict if $G$ is not discrete.

COROLLARY 30. Let $G$ be a locally compact group that is not compact, and let $q<p<\infty$. Then the space of bounded convolution operators from $L^{p}(G)$ to $C^{p, q}(G)$ is just $L_{p, p}^{p, \infty}$, with an equivalent norm.

Proof. This is merely a restatement of Corollary 29.

Observe that we cannot replace the space $C^{p, q}(G)$ by $L_{\text {lar }}^{q}$, in Corollary 30 , because every element of $L_{p}^{r}(G)$, with $r>p$, is a bounded operator from $L^{p}(G)$ to $L_{\mathrm{lar}}^{q}$, and $L_{p}^{r}(G) \not \subset L_{p, p}^{p, \infty}$.

REMARK 16. The method of proof of Theorem 28 can also be used to prove Theorem 27, and to show in addition that, if $G$ is compact, $1 \leqslant p \leqslant 2$, and $q<p$, then

$$
\|T\|_{L_{p, p}^{p, \infty}} \leqslant 2^{1 / p+1 / q} C_{q}\|T\|_{L_{p}^{q}}
$$

for all $T$ in $L_{p}^{q}(G)$. The proofs of Theorem 28 given in [Ste 2], [Saw], [Dos], and [Gar] do not lead to explicit estimates on the norm of $T$ in $L_{p, p}^{p, \infty}$. 
ADDED IN PROOF. Michael Cowling has recently shown that, if $G$ is a semisimple Lie group, with finite centre, and if $1<p<2$, then $L^{p}(G) * L^{2}(G)$ $C L^{2}(G)$; this proves the conjecture made in Remark 9.

\section{REFERENCES}

[Blo] A. P. Blozinski, Convolution of $L(p, q)$ functions, Proc. Amer. Math. Soc. 32 (1972), 237-240. MR 44 \#5724.

[BE] B. Brainerd and R. E. Edwards, Linear operators which commute with translations. I, Representation theorem, J. Austral. Math. Soc. 6 (1966), 289-327. MR 34 \#6542.

[Bri] J. Brillhart, On the Rudin-Shapiro polynomials, Duke Math. J. 40 (1973), 335-353. MR 47 \#3645.

[BB] P. L. Butzer and H. Berens, Semi-groups of operators and approximation, Die Grundlehren der math. Wissenschaften, Band 145, Springer-Verlag, New York, 1967. MR 37 \#5588.

[Cal] A.-P. Calderón, Intermediate spaces and interpolation, the complex method, Studia Math. 24 (1964), 113-190. MR 29 \#5097.

[Coi] R. R. Coifman, Remarks on weak type inequalities for operators commuting with translations, Bull. Amer. Math. Soc. 74 (1968), 710-714. MR 37 \#6687.

[Dos] R. Doss, Some inclusions in multipliers, Pacific J. Math. 32 (1970), 643-646. MR 41 \#5892.

[Ebe] S. E. Ebenstein, $\Lambda(p)$ sets and Sidon sets, Proc. Amer. Math. Soc. 36 (1972), 619-620. MR 46 \#9649.

[EH] R. E. Edwards and E. Hewitt, Pointwise limits for sequences of convolution operators, Acta Math. 113 (1965), 181-218. MR 31 \#1522.

[EP] R. E. Edwards and J. F. Price, $A$ naively constructive approach to boundedness principles, with applications to harmonic analysis, Enseignement Math. (2) 16 (1970), 255296 (1971). MR 45 \#2497.

[EL] P. Eymard and N. Lohoué, Sur la racine carrée du noyau de Poisson dans les espaces symetriques, et une conjecture de E. M. Stein, Ann. Sci. École Norm Sup. 8 (1975), 179-188.

[FS] C. Fefferman and E. M. Stein, $H^{P}$-spaces of several variables, Acta Math. 129 (1972), 137-193.

[F-T] A. Figà-Talamanca, Multipliers of p-integrable functions, Ph. D. Thesis, University of California at Los Angeles, 1964.

[F-T P 1] A. Figà-Talamanca and J. F. Price, Applications of random Fourier series over compact groups to Fourier multipliers, Pacific J. Math. 43 (1972), 431-441. MR 47 \#7330.

[F-T P 2] - Rudin-Shapiro sequences on compact groups, Bull. Austral. Math. Soc. 8 (1973), 241-245. MR 47 \#3908.

[Fou] J. J. F. Fournier, Multipliers of weak type, University of British Columbia, 1973 (ty pescript).

[Gar] A. M. Garsia, Topics in almost everywhere convergence, Lectures in Advanced Math., 4, Markham, Chicago, III., 1970. MR 41 \#5869.

[Gau] G. I. Gaudry, Bad behaviour and inclusion results for multipliers of type (p, q), Pacific J. Math. 35 (1970), 83-94. MR 42 \#6515.

[GI] G. I. Gaudry and I. R. Inglis, Approximation of multipliers, Proc. Amer. Math. Soc. 44 (1974), $381-384$.

[Gil] J. E. Gilbert, $L^{P}$.Convolution operators and tensor products of Banach spaces. I (to appear).

[Gre] F. P. Greenleaf, Invariant means on topological groups and their applications, Van Nostrand Math. Studies, no. 16, Van Nostrand Reinhold, New York, 1969. MR 40 \#4776.

[Her 1] C. S. Herz, The theory of p-spaces with an application to convolution operators, Trans. Amer. Math. Soc. 154 (1971), 69-82. MR 42 \#7833. 
[Her 2] C. S. Herz, Harmonic synthesis for subgroups, Ann. Inst. Fourier (Grenoble) 23 (1973), fasc. 3, 91-125.

[HR] E. Hewitt and K. A. Ross, Abstract harmonic analysis. I, II, Die Grundlehren der math. Wissenschaften, Bänd 115, 152, Springer-Verlag, Berlin and New York, 1963, 1970. MR 28 \#158; 41 \#7378, erratum, 42, p. 1825.

[Hun] R. A. Hunt, On $L(p, q)$ spaces, Enseignement Math. (2) 12 (1966), 249-276. MR 36 \#6921.

[Kah] J.-P. Kahane, Séries de Fourier absolument convergentes, Ergebnisse der Mathematik und ihrer Grenzgebiete, Band 50, Springer-Verlag, Berlin and New York, 1970. MR 43 \#801.

[Kat] Y. Katznelson, An introduction to harmonic analysis, Wiley, New York, 1968. MR 40 \#1734.

[KS] R. A. Kunze and E. M. Stein, Uniformly bounded representations and harmonic analysis of the $2 \times 2$ real unimodular group, Amer. J. Math. 82 (1960), 1-62. MR 29 \#1287.

[Lar] R. Larsen, The multiplier problem, Lecture Notes in Math., vol. 105, SpringerVerlag, Heidelberg and New York, 1969.

[LP] J.-L. Lions and J. Peetre, Sur une classe d'espaces d'interpolation, Inst. Hautes Études Sci. Publ. Math. No. 19 (1964), 5-68. MR 29 \#2627.

[Lip 1] R. L. Lipsman, Uniformly bounded representations of $\operatorname{SL}(2, C)$, Amer. J. Math. 91 (1969), 47-66. MR 39 \#355.

[Lip 2] Uniformly bounded representations of the Lorentz groups, Amer. J. Math. 91 (1969), 938-962. MR 42 \#1946.

[Lip 3] Harmonic analysis on $S L(n, C)$, J. Functional Analysis 3 (1969), 126-155. MR 38 \#5997.

[Lip 4] - An indicator diagram for locally compact unimodular groups, Duke Math. J. 36 (1969), 765-780. MR 41 \#8592.

[Mac] G. W. Mackey, Induced representations of locally compact groups. I, Ann. of Math. (2) 55 (1952), 101-139. MR 13, 434.

[MZ] D. Montgomery and L. Zippin, Topological transformation groups, Interscience, New York, 1955. MR 17, 383. 175-179.

[Obe] D. M. Oberlin, $M_{p}(G) \neq M_{q}(G),\left(p^{-1}+q^{-1}=1\right)$, Israel J. Math. 22 (1975),

[Pee] J. Peetre, A theory of interpolation of normed spaces, Notas de Matemática, no. 39, Instituto de Matemática Pura e Aplicada, Conselho Nacional de Pesquisas, Rio de Janeiro, 1968. MR 39 \#4662.

[Raj 1] M. Rajagopalan, $L^{p}$-conjecture for locally compact groups. 1, Trans. Amer. Math. Soc. 125 (1966), 216-222. MR 34 \#1868.

[Raj 2] $\longrightarrow L^{p}$-conjecture for locally compact groups. II, Math. Ann. 169 (1967), 331-339. MR 34 \#8213.

[Ric] N. W. Rickert, Convolution of $L^{2}$-functions, Colloq. Math. 19 (1968), $301-$ 303. MR 37 \#4509.

[Saw] S. A. Sawyer, Maximal inequalities of weak type, Ann. of Math. (2) 84 (1966), 157-174. MR 35 \#763.

[Sta] J. D. Stafney, Analytic interpolation of certain multiplier spaces, Pacific J. Math. 22 (1970), $241-248$.

[Ste 1] E. M. Stein, Interpolation of linear operators, Trans. Amer. Math. Soc. 83 (1956), 482-492. MR 18, 575.

[Ste 2] On limits of sequences of operators, Ann. of Math. (2) 74 (1961), 140-170. MR 23 \#A2695.

[Ste 3] Singular integrals and differentiability properties of functions, Princeton Math. Series, no. 30, Princeton Univ. Press, Princeton, N. J., 1970. MR 44 \#7280.

[SW 1] E. M. Stein and G. Weiss, An extension of a theorem of Marcinkiewicz and some of its applications, J. Math. Mech. 8 (1959), 263-284. MR 21 \#5888. 
[SW 2] E. M. Stein and G. Weiss, Introduction to Fourier analysis on Euclidean spaces, Princeton Math. Ser., no. 32, Princeton Univ. Press, Princeton, J. J., 1971. MR 46 \#4102.

[Wen] J. G. Wendel, Left centralisers and isomorphisms of group algebras, Pacific J. Math. 2 (1952), 251-261. MR 14, 246.

[Zaf 1] M. Zafran, $A$ multilinear interpolation theorem (to appear).

[Zaf 2] _-Multiplier transformations of weak type, Ann. of Math. (2) 101 (1975), 34-44.

DEPARTMENT OF MATHEMATICS, UNIVERSITY OF BRITISH COLUMBIA, VANCOUVER, BRITISH COLUMBIA, CANADA 